\title{
Comparison of large-scale Birkeland currents determined from Iridium and SuperDARN data
}

\author{
D. L. Green ${ }^{1}$, C. L. Waters ${ }^{1}$, B. J. Anderson ${ }^{2}$, H. Korth ${ }^{2}$, and R. J. Barnes ${ }^{2}$ \\ ${ }^{1}$ School of Mathematical and Physical Sciences and CRC for Satellite Systems, The University of Newcastle, New South \\ Wales, Australia \\ ${ }^{2}$ The Johns Hopkins University Applied Physics Laboratory, Laurel, Maryland, USA
}

Received: 4 November 2005 - Revised: 12 February 2006 - Accepted: 13 March 2006 - Published: 19 May 2006

\begin{abstract}
The Birkeland currents, $J_{\|}$, electrically couple the high latitude ionosphere with the near Earth space environment. Approximating the spatial distribution of the Birkeland currents may be achieved using the divergence of the ionospheric electric field, $\boldsymbol{E}_{\perp}$, assuming zero conductance gradients such that $J_{\|} \approx \Sigma_{\mathrm{p}} \nabla \cdot \boldsymbol{E}_{\perp}$. In this paper, electric field data derived from the Super Dual Auroral Radar Network (SuperDARN) are used to calculate $\Sigma_{\mathrm{p}} \boldsymbol{\nabla} \cdot \boldsymbol{E}_{\perp}$, which is compared with the Birkeland current distribution derived globally from the constellation of Iridium satellites poleward of $60^{\circ}$ magnetic latitude. We find that the assumption of zero conductance gradients is often a poor approximation. On the dayside, in regions where the SuperDARN electric field is constrained by radar returns, the agreement in the locations of regions of upward and downward current between $\Sigma_{\mathrm{p}} \boldsymbol{\nabla} \cdot \boldsymbol{E}_{\perp}$ and $\boldsymbol{J}_{\|}$obtained from Iridium data is reasonable with differences of less than $3^{\circ}$ in the latitudinal location of major current features. It is also shown that away from noon, currents arising from conductance gradients can be larger than the $\Sigma_{\mathrm{p}} \boldsymbol{\nabla} \cdot \boldsymbol{E}_{\perp}$ component. By combining the $\Sigma_{\mathrm{p}} \boldsymbol{\nabla} \cdot \boldsymbol{E}_{\perp}$ estimate in regions of radar coverage with in-situ estimates of conductance gradients from DMSP satellite particle data, the agreement with the Iridium derived $J_{\|}$is considerably improved. However, using an empirical model of ionospheric conductance did not account for the conductance gradient current terms. In regions where radar data are sparse or nonexistent and therefore constrained by the statistical potential model the $\Sigma_{\mathrm{p}} \boldsymbol{\nabla} \cdot \boldsymbol{E}_{\perp}$ approximation does not agree with $J_{\|}$ calculated from Iridium data.
\end{abstract}

Keywords. Ionosphere (Electric fields and currents; Plasma convection; Polar ionosphere)

\section{Introduction}

Electromagnetic power of the order of tens of Giga Watts is continually deposited into the Earth's ionosphere, heating

Correspondence to: D. L. Green

(david.lindsay.green@gmail.com) the polar atmosphere, driving neutral winds, and creating a large system of circulating plasma (Richmond and Thayer, 2000). This transfer of energy is largely driven by the interaction of the interplanetary medium with the magnetic field of the Earth causing field aligned currents to flow between the magnetosphere and ionosphere which close in the auroral ionosphere (Kelley, 1989). The Birkeland currents convey stress between the solar wind and magnetosphere to the ionosphere (Cowley, 2000). Satellite observations (e.g., Zmuda et al., 1966) confirmed Birkeland's ideas of high altitude field-aligned currents (Birkeland, 1908) and due to their importance in electrically coupling near Earth space with the ionosphere, the determination of the large-scale structure of the Birkeland currents is central to understanding the energetics and dynamics of the magnetosphere-ionosphere system.

The morphology of the large-scale Birkeland currents has been inferred using ground magnetometer data (Kamide et al., 1981; Richmond and Kamide, 1988), ionosphere plasma convection (Sofko et al., 1995), and in situ satellite measurements (Iijima and Potemra, 1976; Zanetti et al., 1983; Weimer, 2000; Eriksson et al., 2002). The development and expansion of the Super Dual Auroral Radar Network (SuperDARN) has provided a tool capable of monitoring large-scale ionospheric plasma convection (Greenwald et al., 1985). SuperDARN employs over-the-horizon radar technology to probe the high latitude ionosphere by detecting backscatter of the HF signal modified by plasma density irregularities. In common mode, the radars scan through 16 successive azimuthal directions or beams spaced by $3.3^{\circ}$ with an integration time of $7 \mathrm{~s}$ per beam (Ruohoniemi and Baker, 1998). Assuming zero (or small) horizontal gradients in the height-integrated ionospheric conductivity (hereafter referred to as conductance), SuperDARN measurements potentially provide high time resolution (2 min) spatial maps of the Birkeland currents (Sofko et al., 1995; Kustov et al., 2000; McWilliams et al., 2001). In the present paper we extend these studies using the maps of plasma convection provided as a SuperDARN data product to determine the extent

Published by Copernicus GmbH on behalf of the European Geosciences Union. 
to which these convection patterns combined with conductance estimates yield the Birkeland currents recorded by low altitude satellite magnetometers.

Despite the different ways the Birkeland currents may be observed, mapping the global distribution with both high spatial and temporal resolution without significant reliance on empirical models has only recently become possible. In the past, single satellite studies applied statistical averaging of multiple orbits resulting in time averaged, global pictures of the Birkeland current distribution (Iijima et al., 1984; Stauning, 2002). Multi-satellite systems such as the Cluster spacecraft allow calculation of the instantaneous, spatial distribution but only within a limited field of view (Amm, 2002; Dunlop et al., 2002). Application of the Assimilative Mapping of Ionospheric Electrodynamics (AMIE) method (Richmond and Kamide, 1988) allows for $\sim 2$ min time resolution estimates of the global Birkeland current pattern by constraining statistical models of ionospheric parameters. However, the reliance on ionospheric conductance estimates in AMIE motivates the search for independent estimates of ionospheric parameters.

The conductance of the ionosphere is a pivotal parameter in the Birkeland current circuit. The large scale size of this system in the field aligned direction allows the altitude variation of conductance to be replaced by height integrated Pedersen $\left(\Sigma_{\mathrm{P}}\right)$ and Hall $\left(\Sigma_{\mathrm{H}}\right)$ conductances. As a first approximation, the horizontal variation in conductance can also be ignored and there have been a number of studies to determine the validity of assuming uniform ionospheric conductance. The Birkeland currents derived from radar data, assuming no horizontal conductance gradients, were compared with images of the aurora obtained by the visible imaging system (VIS) Earth Camera and the Far Ultraviolet Imager (UVI) on the Polar spacecraft by McWilliams et al. (2001). For their event, the upward Birkeland current regions were co-located with bright aurora. Kosch et al. (2001) combined radar data, ground based magnetometer observations and used a fixed conductance ratio, $\Sigma_{\mathrm{H}} / \Sigma_{\mathrm{P}}=1.1$, to estimate the ionospheric conductance distribution over the radar fields of view. Their results for nightside regions (23:0024:00 MLT) demonstrated that conductance gradients cannot be ignored, even for quiet geomagnetic conditions.

The contribution to field-aligned currents from conductance gradients in the morning sector was examined by Sato et al. (1995). They used a combination of the KRM (Kamide et al., 1981) and AMIE (Richmond and Kamide, 1988) techniques. Defence Meteorological Satellite Program (DMSP) data and electron densities derived from EISCAT data were used to show that the relative magnitude of the field-aligned current component associated with conductance gradients compared with the total field-aligned currents varies with latitude and that these currents may even flow in opposite directions. Additionally, a mesoscale morning sector study of ionospheric electrodynamics by Amm et al. (2005) combined ionospheric conductance data derived from UVI and PIXIE instruments on the Polar satellite with ground-based electromagnetic data from the MIRACLE network. They found that the overwhelming part of the field-aligned currents are those associated with gradients in ionospheric conductance.

Electric field estimates over all local times from $60^{\circ}$ to the pole have become a standard SuperDARN data product. If one also knew the ionospheric conductances one could evaluate the energetics of the magnetosphere-ionosphere interaction. The accuracy of the convection and conductances are difficult to assess so it is hard to judge the reliability of such calculations. However, the $J_{\|}$distribution could also be obtained from the convection and conductances and comparing the $J_{\|}$inferred from convection against Birkeland currents obtained by in-situ observations on a global scale provides a comprehensive test of the accuracy and reliability of the convection-conductance approach. In this paper we report the first such direct comparisons of independently derived Birkeland current distributions. The magnetic field measurements from the Iridium satellite constellation (Anderson et al., 2000) provide estimates of the Birkeland currents (Waters et al., 2001). While the minimum integration time required by Iridium $(>45 \mathrm{~min}$ ) is larger than that of the radar data $(\sim 2 \mathrm{~min})$, here we compare the results from the two techniques for intervals when the spatial distribution of the currents is relatively constant for $\sim 1 \mathrm{~h}$. These data provide an independent check on the accuracy of the SuperDARN derived Birkeland current estimates. In order to achieve this, the method for estimating the Birkeland currents from Iridium data, as described by Waters et al. (2001) requires some modifications and these are described in Sect. 3. Comparisons between the Birkeland current distribution as derived from SuperDARN and Iridium data are presented in Sect. 4. Finally, the results are discussed in Sect. 5 where we focus on the difficulties in determining ionospheric conductance and how this affects estimates of the global Birkeland current patterns.

\section{Birkeland Currents from SuperDARN}

SuperDARN provides estimates of plasma convection velocity, $\boldsymbol{v}=\boldsymbol{E} \times \boldsymbol{B} / B^{2}$, in the F-region ionosphere with respect to the geomagnetic field $(\boldsymbol{B})$, allowing the ionospheric electric field to be determined (Greenwald et al., 1995; Ruohoniemi and Baker, 1998) in the Earth's reference frame. The received radar backscatter depends on geomagnetic conditions and typically covers $10-60 \%$ of the probed region (Greenwald et al., 1995) allowing a global picture of the 2D ionospheric electric field, $\boldsymbol{E}_{\perp}$, to be inferred (Shepherd and Ruohoniemi, 2000). Since the global ionospheric plasma convection and Birkeland current patterns orientate with magnetospheric processes while the Earth rotates beneath, all calculations in this paper assume an Earth centered, non-rotating frame of reference. The electric field values derived from 
SuperDARN were transformed into this Earth centered, nonrotating reference frame.

On time scales greater than seconds, the total ionospheric current, $\boldsymbol{J}$, is solenoidal, i.e. $\boldsymbol{\nabla} \cdot \boldsymbol{J}=0$ (Kelley, 1989; Richmond and Thayer, 2000). Assuming radial field lines, the total current consists of field aligned Birkeland currents $\left(\hat{\boldsymbol{r}} \cdot \boldsymbol{J}=J_{\|}\right)$and ionospheric surface currents $\left(\boldsymbol{J}_{\perp}\right)$ that can be assumed to flow horizontally in a thin spherical shell that approximates the ionosphere (Backus, 1986; Cowley, 2000). The radial current density is related to the surface currents by

$$
\nabla \cdot J_{\perp}=J_{\|}
$$

$J_{\perp}$ consists of Hall and Pedersen components such that

$$
\boldsymbol{J}_{\perp}=\Sigma_{\mathrm{P}} \boldsymbol{E}_{\perp}+\Sigma_{\mathrm{H}} \hat{\boldsymbol{B}} \times \boldsymbol{E}_{\perp}
$$

where $\Sigma_{\mathrm{H}}$ and $\Sigma_{\mathrm{P}}$ are the height-integrated Hall and Pedersen conductivities respectively and $\hat{\boldsymbol{B}}$ is the unit vector in the direction of the geomagnetic field. Combining Eqs. (1) and (2) gives (Untiedt and Baumjohann, 1993)

$$
J_{\|}=\Sigma_{\mathrm{P}} \nabla \cdot \boldsymbol{E}_{\perp}+\boldsymbol{E}_{\perp} \cdot \nabla \Sigma_{\mathrm{P}}+\nabla \Sigma_{\mathrm{H}} \cdot \hat{\boldsymbol{B}} \times \boldsymbol{E}_{\perp}
$$

Equation (3) shows that $J_{\|}$consists of two current sources. The first are those associated with a divergence in the ionospheric electric field, $J_{\nabla \cdot E}$, and are represented by the $\Sigma_{\mathrm{P}}$ $\boldsymbol{\nabla} \cdot \boldsymbol{E}_{\perp}$ term. The other terms describe currents due to gradients in ionospheric conductance, $J_{\nabla \Sigma}$. The divergence of the electric field can be regarded as the div-E current per unit Pedersen conductance. Taking only the first term from the right hand side of Eq. (3) and denoting the div-E current as $J_{\nabla \cdot E}$, one has

$$
\nabla \cdot \boldsymbol{E}_{\perp}=\frac{J_{\nabla \cdot E}}{\Sigma_{\mathrm{P}}}
$$

This is equivalent to the analysis of Sofko et al. (1995) where $J_{\nabla \cdot E}$ was developed in terms of the plasma velocity observed by SuperDARN such that

$$
\nabla \cdot E_{\perp}=\frac{J_{\nabla \cdot E}}{\Sigma_{\mathrm{P}}}=\boldsymbol{B} \cdot \nabla \times \boldsymbol{v}
$$

The div-E currents, $J_{\nabla \cdot E}$, may be estimated from SuperDARN observations while the total Birkeland current, $J_{\|}$, consisting of both sources can be determined from Iridium data.

\section{Calculation of Birkeland currents from Iridium}

\subsection{Modelling of $J_{\|}$}

Engineering magnetometer data from the Iridium satellite constellation have been processed as described by Anderson et al. (2000) giving the cross-track component of the Birkeland current induced, full vector magnetic perturbation, $\Delta \boldsymbol{b}$.
The magnetic field measurements from over 70 , polar orbiting ( $780 \mathrm{~km}$ altitude) satellites provide maps of the Birkeland currents with a latitudinal resolution of $\sim 3^{\circ}$ for a data accumulation interval of $1 \mathrm{~h}$. The in-situ nature of the Iridium observations provides the only means for calculating 2-D global Birkeland current maps on these times scales without relying on empirical models or assumptions concerning ionospheric conductance. The satellite $\Delta \boldsymbol{b}$ observations are mapped to an ionospheric altitude $(110 \mathrm{~km})$ by applying a correction factor $\left(r / R_{\text {iono }}\right)^{3 / 2}$, where $r$ is the satellite geocentric distance and $R_{\text {iono }}$ is the ionosphere radius.

Methods for determining the large-scale Birkeland currents from satellite $\Delta \boldsymbol{b}$ data without assumptions about the current geometry have been presented by Olsen (1997), Weimer (2000) and Waters et al. (2001). Olsen (1997) used properties of the Laplacian evaluated over spherical surfaces to calculate the field-aligned currents over the entire sphere from time averaged Magsat data. Extensions to the method presented by Waters et al. (2001) that allow eigenvalues of the Laplacian to be used in calculating Birkeland currents from Iridium $\Delta \boldsymbol{b}$ data confined to a spherical cap have been developed and are described here. At Iridium orbit altitudes, the magnetic field is not curl free due to the presence of Birkeland currents. The appropriate representation of $\Delta \boldsymbol{b}$ was reviewed by Backus (1986) and involves the decomposition of vector fields on spherical surfaces into poloidal and toroidal components. The radial currents

$$
\mu_{0} J_{\|} \hat{\boldsymbol{r}}=\nabla \times(\boldsymbol{r} \times \nabla p)
$$

produce toroidal magnetic perturbations,

$$
\triangle \boldsymbol{b}=\boldsymbol{r} \times \nabla Q
$$

where $p$ and $Q$ are the poloidal current and toroidal magnetic scalar functions respectively. As discussed by Waters et al. (2001), shifting to a spherical coordinate system centered on the intersection point of the Iridium satellite paths (Iridium system) allows the available cross-track component of $\Delta \boldsymbol{b}$ to be approximated as azimuthal (east), i.e., $\hat{\boldsymbol{\phi}} \cdot \Delta \boldsymbol{b}$. While the spherical harmonic fitting computations were performed in the Iridium system, the data are presented in geomagnetic coordinates. Expanding the azimuthal component as a linear combination of spherical harmonics, $Y_{l}^{m}(\theta, \phi)$, with coefficients $a_{l}^{m}$ allows calculation of $Q$ according to

$$
\hat{\boldsymbol{\phi}} \cdot \Delta \boldsymbol{b}=\sum_{l=1}^{L} \sum_{m=0}^{M \leq l} a_{l}^{m} \frac{\partial}{\partial \theta} Y_{l}^{m}(\theta, \phi)
$$

and

$$
Q=\sum_{l=1}^{L} \sum_{m=0}^{M \leq l} a_{l}^{m} Y_{l}^{m}(\theta, \phi)
$$

Backus (1986) showed that an expression for the radial currents in terms of the $a_{l}^{m}$ coefficients may be obtained by 
combining the toroidal representation of $\Delta \boldsymbol{b}$ in Eq. (7) with Ampere's law to give

$$
\begin{aligned}
\mu_{0} r J_{\|} & =\boldsymbol{r} \cdot \nabla \times \triangle \boldsymbol{b} \\
& =\nabla^{2} Q .
\end{aligned}
$$

Using the identity for spherical harmonics,

$$
\nabla^{2} Y_{l}^{m}(\theta, \phi)=-l(l+1) Y_{l}^{m}(\theta, \phi)
$$

the radial currents are (Backus, 1986)

$$
\mu_{0} r J_{\|}=-\sum_{l=1}^{L} l(l+1) \sum_{m=0}^{M \leq l} a_{l}^{m} Y_{l}^{m}(\theta, \phi)
$$

The south $(\hat{\boldsymbol{\theta}})$ component of $\Delta \boldsymbol{b}$ may be reconstructed using

$$
\hat{\boldsymbol{\theta}} \cdot \Delta \boldsymbol{b}=-\frac{1}{\sin \theta} \sum_{l=1}^{L} \sum_{m=0}^{M \leq l} a_{l}^{m} \frac{\partial}{\partial \phi} Y_{l}^{m}(\theta, \phi) .
$$

Eqation (13) is not required for the calculation of $J_{\|}$since the $a_{l}^{m}$ coefficient set completely defines $Q$. The inverse problem of calculating $a_{l}^{m}$ from the measurements, $\hat{\boldsymbol{\phi}} \cdot \Delta \boldsymbol{b}$, is achieved using a least squares formulation and a singular value decomposition algorithm as discussed in Waters et al. (2001). Modifications to the process of obtaining the global configuration of the currents take advantage of Eq. (12) and are described in the next section.

\subsection{Numerical evaluation of $J_{\|}$}

Since Birkeland current closure occurs within the high latitude polar regions our analysis of magnetic perturbation data is restricted to a spherical cap whose equatorward boundary is a function of geomagnetic activity. Spherical Cap Harmonic Analysis (SCHA) described by Haines $(1985,1988)$ is used as the basis of the numerical approach. Haines showed that to model a vector field over a spherical cap the scalar potential function ( $Q$ in this case) should only be constructed from spherical harmonic functions that satisfy one of two boundary conditions at the cap edge $\left(\theta_{c}\right)$. These two boundary conditions are

$$
\begin{aligned}
Y_{n}^{m}\left(\theta_{c}, \phi\right) & =0 \\
\frac{\partial}{\partial \theta} Y_{n}^{m}\left(\theta_{c}, \phi\right) & =0 .
\end{aligned}
$$

Using both sets of basis functions allows the scalar potential to have zero and non-zero values at the pole and cap boundary, respectively. The complication with this approach is that the spherical harmonics that satisfy these boundary conditions are usually of non-integral degree $(n)$ but integral order $(m)$. Since the values of $n$ that satisfy Eq. (14) depend on $m$, Haines (1985) denoted the degree by $n_{k}(m)$, where $k$ is an integer that can be used much like $l$ in the full sphere spherical harmonics for defining the latitudinal resolution of the functions. Numerical calculation of the non-integral functions becomes increasingly difficult as $k$ increases due to limitations in computational precision (Haines, 1988). The algorithm provided by Haines for the calculation of non-integral Legendre functions requires special extended precision hardware or a reduction in accuracy when computing $k>12 \mathrm{Leg}$ endre functions. Using a capsize of $\theta_{c}=50^{\circ}$ (colatitude) and a $k$ value of 12 yields a latitudinal resolution of $8.4^{\circ}$ while a $k$ value of 30 corresponds to a latitudinal resolution of $3.1^{\circ}$.

In order to calculate the associated Legendre functions with the required latitudinal resolution on a standard personal computer (53 bit mantissa) a new algorithm for calculating non-integral spherical harmonics was implemented, employing the multi precision hypergeometric function from the Cephes Mathematical Library (Moshier, 1995). Extending the work of Hobson (1955), analytic expressions for the non-integral associated Legendre functions and their latitudinal derivatives were derived and are given in Appendix A. This new algorithm avoids computer accuracy limitations by controlling the precision of the calculations at the software level which allows very high order, non-integral, Associated Legendre functions to be generated.

Using the two sets of functions that satisfy Eq. (14) in the construction of $Q$ allows the along track component of $\Delta \boldsymbol{b}$, that is, the north-south component in the Iridium system, to have any magnitude at the cap boundary that satisfies $\nabla \cdot \Delta \boldsymbol{b}=0$. However, since only cross-track data are used in the fitting process the reconstructed $\Delta \boldsymbol{b}$ may exhibit unphysical features in the south component and still be divergenceless. To avoid this problem the SCHA was modified according to the suggestion of Haines (1988). The construction of the scalar potential, $Q$, was limited to one set of spherical harmonic functions. Using only the set of functions that satisfy Eq. (14a) additionally constrains the south component of the reconstructed $\Delta \boldsymbol{b}$ to be zero at the cap boundary while still allowing arbitrary non-zero azimuthal values. This approach may contaminate the global reconstructed field or potential only if non-zero $\hat{\boldsymbol{\theta}} \cdot \Delta \boldsymbol{b}$ values close to the cap boundary are included in the fitting process. Such a case is possible when the full-vector DMSP and Oersted data are included. By examining a worst case SCHA fit where a spherical harmonic satisfying Eq. (14b) is constructed using only functions that satisfy Eq. (14a), the authors have found that possible contamination due to an unrealistic boundary condition is confined to within one wavelength of the maximum $n_{k}(m)$ used in the fit from the cap boundary. For $\mathrm{K}=15$ and a cap boundary at $50^{\circ}$ colatitude this is $\sim 7.4^{\circ}$. Therefore, when fitting the Iridium data the cap boundary is set at a latitude equatorward of the currents determined by examination of the cross-track $\Delta \boldsymbol{b}$ magnitudes. This approach has added advantages that all basis functions are orthogonal and only half the number of coefficients need be calculated (Haines, 1988). Since a latitudinal resolution similar to that obtained 
using both sets of functions is achieved when using only one set, the $k$ value only needs to be half that required when using both sets. Hereafter all $k$ values are associated with a single set of basis functions.

An alternative and simpler method is to stretch the $\Delta \boldsymbol{b}$ data coordinates to cover a hemisphere and use what de Santis (1992) has named Adjusted Spherical Harmonic Analysis (ASHA). This involves scaling the data and then using the integral spherical harmonic functions to determine $a_{l}^{m}$ followed by a rescaling of the reconstructed field. This method is used to obtain the global convection maps from the SuperDARN data (Ruohoniemi and Baker, 1998). While this approach gives the correct reconstructed vector field, such as $\Delta \boldsymbol{b}$, stretching the data in this way distorts the basis functions so that they are no longer eigenfunctions of the Laplacian (see Eq. 10). Therefore, without determining the scaling functions required to relate SCHA to ASHA coefficients, ASHA cannot be used to calculate the currents from $\hat{\boldsymbol{\phi}} \cdot \Delta \boldsymbol{b}$ data alone. One must obtain the full vector $\Delta \boldsymbol{b}$ and then numerically apply Ampere's law to obtain the currents. To avoid this unnecessary numerical differentiation and to ensure that all of the useful information is fully represented in the harmonic coefficients, we adopted the approach of Haines (1988) and the algorithms we have developed take advantage of the eigenfunction/eigenvalue properties of the spherical harmonics over a spherical cap, enabling analytical representation of both $\Delta \boldsymbol{b}$ and $J_{\|}$with high latitudinal resolution on a standard personal computer.

\section{Results}

At present, the availability of data from Iridium is around 1 sample per $200 \mathrm{~s}$ for each satellite. This limits the spatial and temporal resolution of the Birkeland currents calculated from these data. In previous work, results of the fitting process were validated against full vector magnetometer data from DMSP or Oersted spacecraft (Waters et al., 2004, H. Korth.: private communication, 2005). For our analysis we use magnetic field data from the Iridium system as well as data from the DMSP F13, F15 and Oersted satellites. Because the Iridium magnetic field data are bundled in the satellite engineering packet, the time sampling of data sent to the ground is limited by the rate at which the large volume of engineering data can be transmitted. In this configuration, the interval between telemetered magnetic field samples is around one sample every $200 \mathrm{~s}$ for each satellite. It is therefore desirable to supplement these data with higher time resolution magnetic field data obtained by other low Earth orbiting satellites. For the results presented in this paper, we have included the full vector magnetic perturbation data from the DMSP and Oersted satellites in the fitting process. Data from the DMSP and Oersted spacecraft were provided with $1 \mathrm{~Hz}$ sample rate. Since the Iridium data are sampled at a much slower rate, the DMSP and Oersted data must be weighted

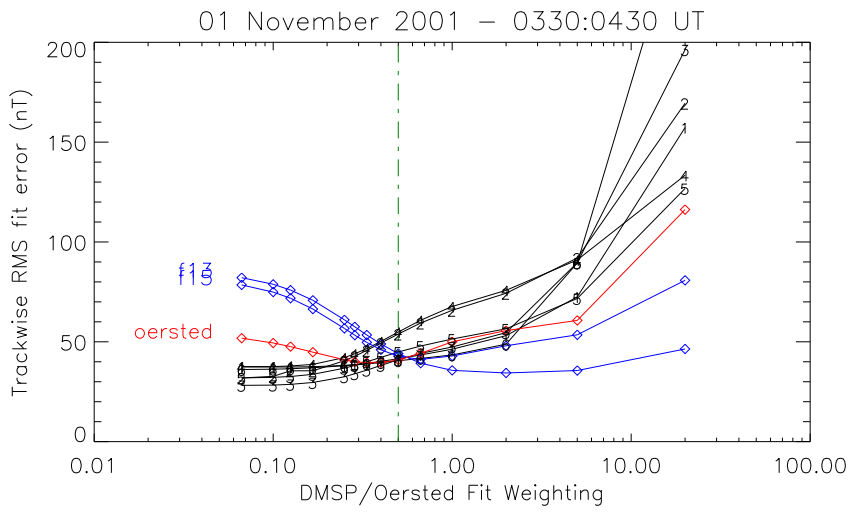

Fig. 1. Trackwise plot of the azimuthal fit error for all six Iridium tracks (black), two DMSP satellites (blue) and the Oersted satellite (red) as a function of the DMSP and Oersted data weighting factor. The green vertical line indicates the chosen weighting factor for this event.

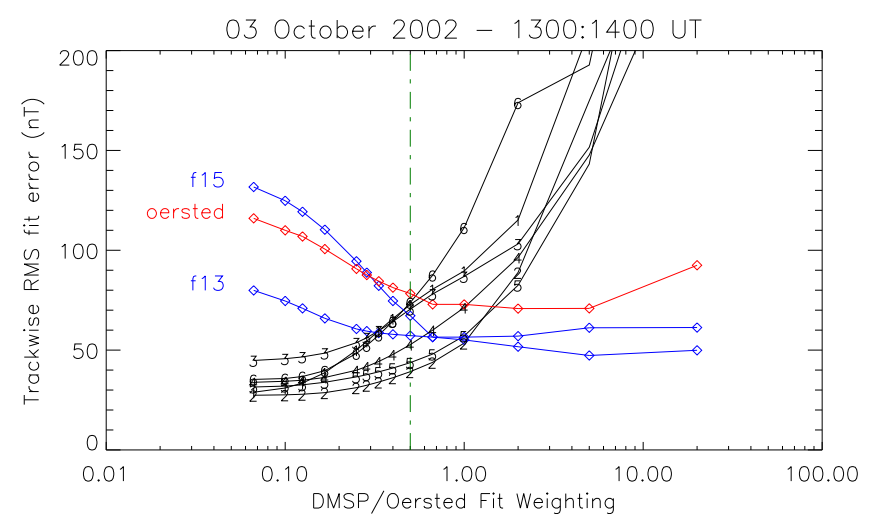

Fig. 2. Trackwise plot of the azimuthal fit error for all six Iridium tracks (black), two DMSP satellites (blue) and the Oersted satellite (red) as a function of the DMSP and Oersted data weighting factor. The green vertical line indicates the chosen weighting factor for this event.

so that the Iridium data still contributes in the fitting process. The appropriate weighting factor was determined by examining the root mean square (RMS) of the differences between the observed Iridium, DMSP and Oersted data and the resulting $\hat{\boldsymbol{\phi}} \cdot \Delta \boldsymbol{b}$ reconstruction for each track as a function of weighting factor. This fit error is shown for the presented events in Figs. 1 and 2 for the 6 Iridium planes, 2 DMSP tracks and the single Oersted pass. For both events the error in the fit to the Iridium data increases as the weighting factor increases past unity. The weighting factor was chosen to be 0.5 as indicated by the vertical line in these figures.

Selection of events for comparison of SuperDARN with Iridium data is somewhat restricted. The Birkeland current configuration must remain relatively constant throughout the integration time required to collect sufficient Iridium data to attain the required latitudinal resolution. These 


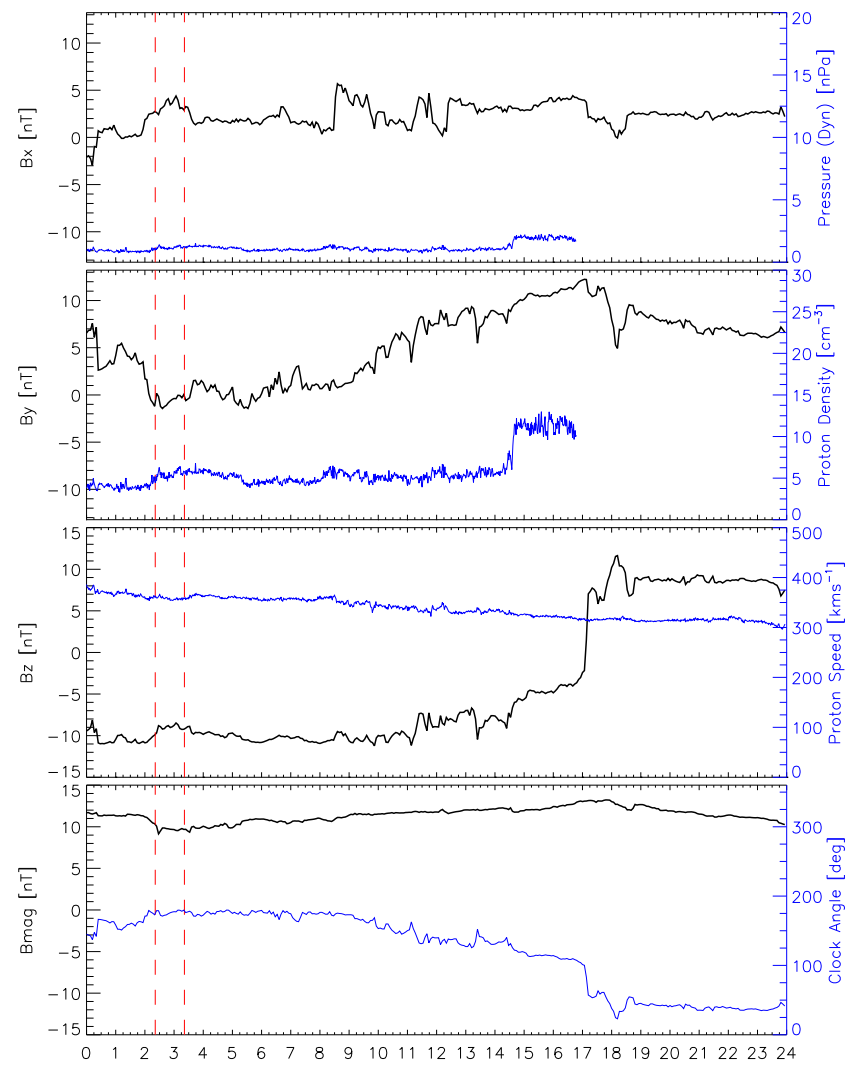

Fig. 3. ACE solar wind parameters showing several hours either side of 02:20-03:20 UT.

$\triangle \boldsymbol{b}$ observations must be of sufficient magnitude to resolve a clear current structure for comparison with SuperDARN results. A constant Birkeland current configuration is typically chosen when (i) the solar wind and IMF conditions are stable (H. Korth: private communication, 2005), (ii) the observed SuperDARN ionospheric plasma convection is stable, and (iii) the accumulated Iridium $\triangle \boldsymbol{b}$ observations are self consistent. SuperDARN must receive radar returns covering a large percentage of the probed area. For our analysis we also need DMSP and Oersted data to supplement the Iridium data, but as the coverage for these data is generally excellent, this is not a significant constraint on event selection. More than a dozen events were identified that satisfy these criteria between January 2001 and April 2003. Comparison of the div-E and satellite derived Birkeland currents was performed for all of these events with two representative cases being chosen for this paper.

\subsection{Event 1: 1 November 2001, 03:30-04:30 UT}

The first event illustrates results obtained with radar returns on the nightside in the absence of solar illumination. The interval is 03:30-04:30 UT on 1 November
2001. The interplanetary data from the Advanced Composition Explorer (ACE) satellite and SuperDARN convection maps were examined for stability over the time period. The upstream solar wind data observed by ACE during the interval 02:20-03:20 UT (70 min delay) is shown in Fig. 3. Analysis of the parameters showed a relatively constant southward IMF and the following mean characteristics: $B_{\mathrm{x}}=3.6 \pm 0.6 \mathrm{nT}, B_{\mathrm{y}}=-0.6 \pm 0.5 \mathrm{nT}, B_{\mathrm{z}}=-9.1 \pm 0.4 \mathrm{nT}$, $B_{\mathrm{t}}=9.7 \pm 0.3 \mathrm{nT}$, clock angle of $184 \pm 3^{\circ}, \mathrm{N}_{\mathrm{p}}=5.5 \pm 0.4 \mathrm{~cm}^{-3}$, $\mathrm{v}_{\mathrm{p}}=359 \pm 3 \mathrm{kms}^{-1}$ and $\mathrm{P}_{\mathrm{dyn}}=1.2 \pm 0.1 \mathrm{nPa}$. Throughout the interval the SuperDARN convection maps showed a typical two cell convection pattern consistent with a southward IMF. These are conditions for a stable, global Birkeland current pattern over the event time frame.

The analysis results and comparisons are shown in Figs. 4 through Fig. 9. Figure 4 shows the data included in the SCHA process. A cap size of $\theta_{c}=50^{\circ}$ (colatitude) was used with $k=15$ and $m=5$ yielding a latitudinal resolution of $3.1^{\circ}$. The DMSP (blue) and Oersted (red) data are the full horizontal $\Delta \boldsymbol{b}$ vectors while the Iridium data (black) are the cross track component magnetic perturbations. Figure 5 shows the full vector reconstruction of $\triangle \boldsymbol{b}$ (black) using SCHA described in Sect. 3. Two vortices are visible, one centered on 70 ${ }^{\circ}$ and 07:00 MLT which is associated with a downward current and the other at $66^{\circ}$ from 19:00 to 02:00 MLT associated with an upward current. The resultant Birkeland current pattern is shown in Fig. 6. In the morning sector Region 1 (downward/blue) and Region 2 (upward/red) current systems with an up-down current boundary at $65^{\circ}$ and 08:00 MLT are clearly seen. In the pre-midnight region the R1/R2 up-down current boundary appears at $64^{\circ}, 21: 00$ MLT.

Data from all SuperDARN radars operating in the Northern Hemisphere were used to determine the plasma convection according to the procedure described by Ruohoniemi and Baker (1998). The electric field, $\boldsymbol{E}_{\perp}$, and the corresponding electric potential are shown in Fig. 7 for data averaged over 03:30-04:30 UT. Radar returns were mostly seen on the nightside for this case and, as with all SuperDARN observations, latitudinal coverage is restricted to regions poleward of $60^{\circ}$.

We now compare $J_{\|}$from satellite data (Fig. 6) and $J_{\nabla \cdot E} / \Sigma_{\mathrm{P}}$ from the radar data (Fig. 8). Although we expect the magnitudes of $J_{\|}$and $J_{\nabla \cdot E} / \Sigma_{\mathrm{P}}$ to be different, the spatial structure and location of up-down current boundaries in $J_{\nabla \cdot E} / \Sigma_{\mathrm{P}}$ should resemble those of $J_{\|}$in regions where conductance gradients are small. Figure 8 shows $J_{\nabla \cdot E} / \Sigma_{\mathrm{P}}$ in the shaded colour format with the contours of $J_{\|}$overlayed. Only the significant $J_{\|}$features are used for comparison. These are the R1/R2 current system in the morning sector and the $\mathrm{R} 1 / \mathrm{R} 2$ system in the pre-midnight region

In the morning sector, $J_{\nabla \cdot E} / \Sigma_{\mathrm{P}}$ shows a Region 1 current (downward/blue) at $73^{\circ}$ and 06:00 MLT that extends poleward to $77^{\circ}$ at 08:00 MLT. No significant Region 2, $J_{\nabla . E} / \Sigma_{\mathrm{P}}$ current can be seen in the morning sector. Comparison with the overlayed $J_{\|}$contours shows the peak in $J_{\nabla \cdot E} / \Sigma_{\mathrm{P}}$ 


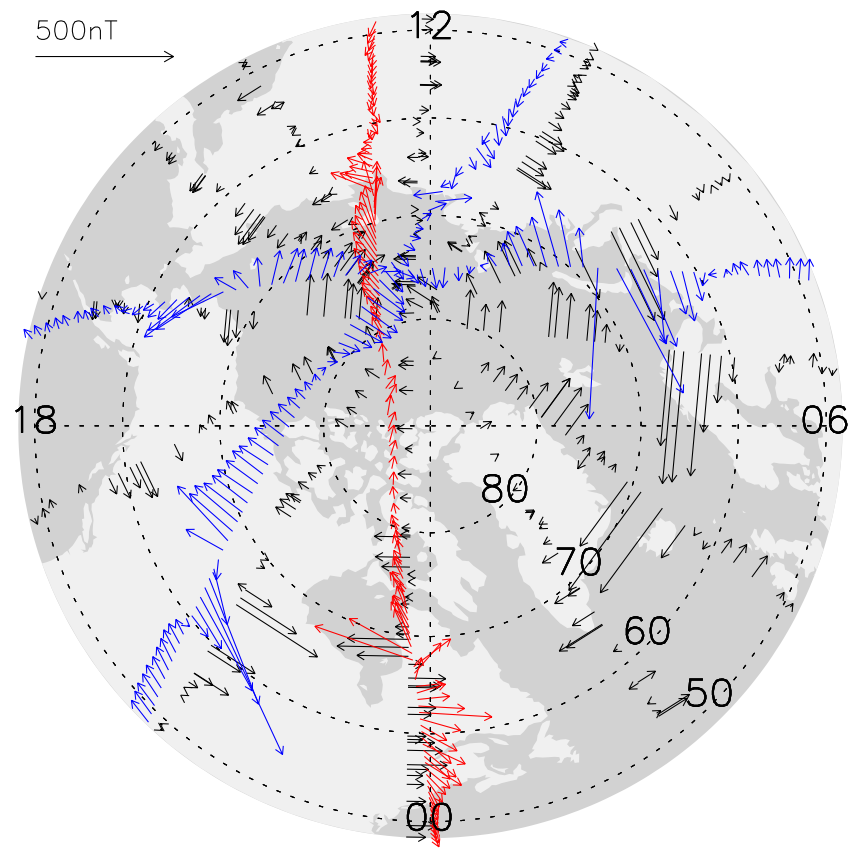

Fig. 4. Cross-track component of the Iridium $\Delta \boldsymbol{b}$ data (black) for 1 November 2001, 03:30-04:30 UT. The two blue tracks are full vector $\triangle \boldsymbol{b}$ observations from DMSP satellite F13 (17:0007:00 MLT track) and F15 for 03:28-03:57 UT and 03:5104:22 UT, respectively. The red track shows full vector $\Delta \boldsymbol{b}$ observations from the Oersted satellite for 0339-0408 UT. Latitudinal coordinates are in the AACGM system.

Region 1 current corresponds to the $J_{\|}$Region 1 maximum $3^{\circ}$ equatorward at $70^{\circ}$ and 06:00 MLT. However, the $J_{\|}$, Region 1 current extends towards noon and shifts equatorward while the $J_{\nabla \cdot E} / \Sigma_{\mathrm{P}}$, Region 1 extends towards noon and shifts poleward. The Region 2 current shown by the $J_{\|}$contours is not reproduced in $J_{\nabla \cdot E} / \Sigma_{\mathrm{P}}$.

In the afternoon sector $J_{\nabla \cdot E} / \Sigma_{\mathrm{P}}$ shows a clear R1/R2 system with an up-down current boundary at $65^{\circ}$ and 18:00 MLT. However, with respect to $J_{\nabla \cdot E} / \Sigma_{\mathrm{P}}$, the $J_{\|} \mathrm{R} 1 / \mathrm{R} 2$ system is $\sim 4^{\circ}$ equatorward and shifted $\sim 3 \mathrm{~h}$ towards midnight. This results in the $J_{\|}$Region 1 current being colocated with the $J_{\nabla \cdot E} / \Sigma_{\mathrm{P}}$ Region 2 current such that they have opposite directions. In addition to these currents, $J_{\nabla \cdot E} / \Sigma_{\mathrm{P}}$ seems to show a large R1/R2 type system between 12:00 and 15:00 MLT with an up-down boundary at $61^{\circ}$. This feature is not seen in $J_{\|}$.

The current density per unit Pedersen conductance, $J_{\nabla \cdot E} / \Sigma_{\mathrm{P}}$, is the div-E component of the Birkeland currents that are estimated from the SuperDARN electric field data, ignoring currents resulting from horizontal conductance gradients. We can compare $J_{\nabla \cdot E} / \Sigma_{\mathrm{P}}$ (by Eq. 4 ) with a full current solution derived from the radar data by combining the SuperDARN electric field values with a conductance model. We shall identify the currents obtained from the SuperDARN electric fields that include the conductance gradient terms



Fig. 5. Reconstructed full vector $\Delta \boldsymbol{b}$ fit as a result of applying SCHA to all data in Fig. 4. Overlays of the DMSP and Oersted magnetic field observations are shown for comparison.

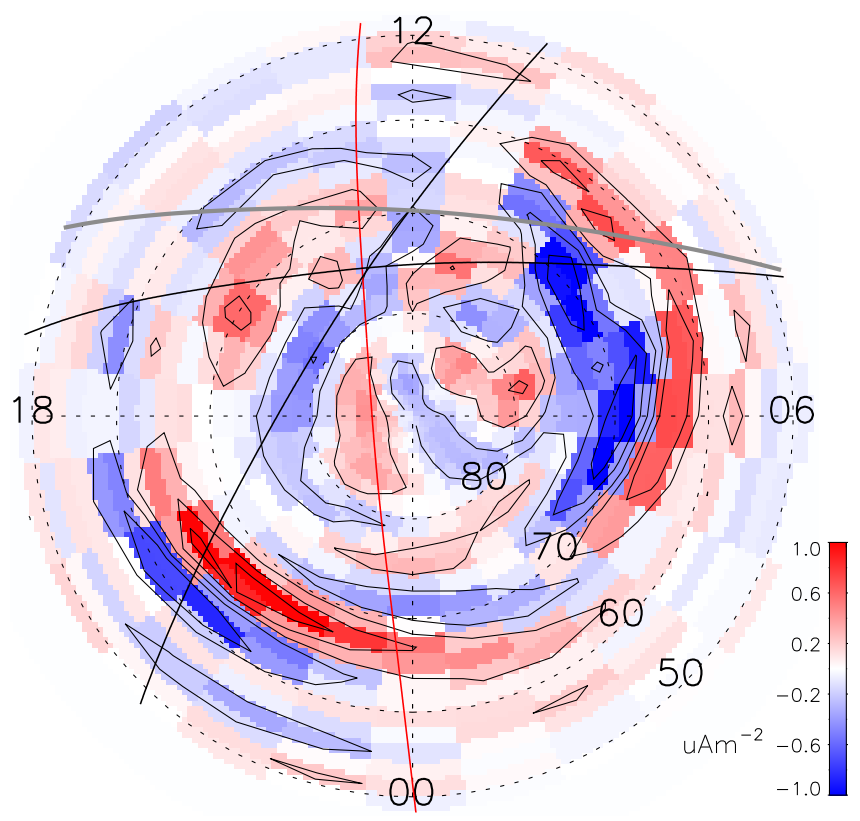

Fig. 6. Birkeland currents, $J_{\|}$derived from the data in Fig. 4 according to Eq. (12) for 03:30-04:30 UT, 1 November, 2001. The DMSP and Oersted tracks are reproduced while the thicker, grey solid line from 06:00 to 18:00 MLT indicates the sunlight terminator boundary in the ionosphere. 


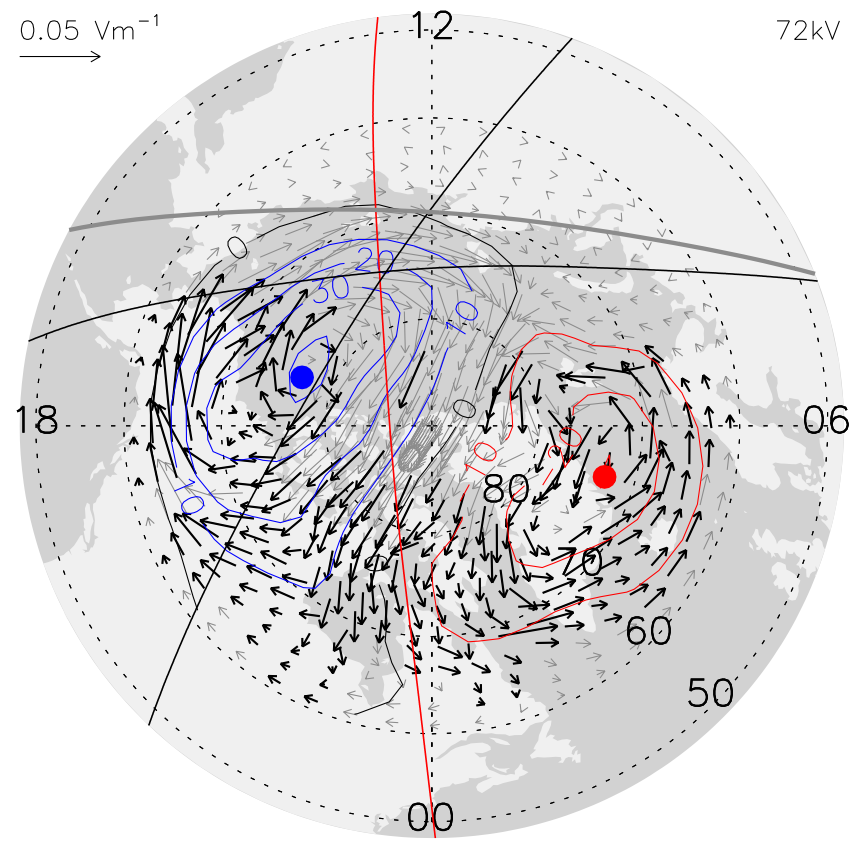

Fig. 7. Electric field vectors (rotated $90^{\circ}$ counter clockwise) calculated from SuperDARN data averaged over 03:30-04:30 UT, 1 November, 2001. The electric potential contours, DMSP and Oersted tracks and the sunlight terminator are overlayed. The extremes in potential are located at the blue (-ve) and red (+ve) dots. The electric field vectors are bold at locations where radar returns were received.

from the conductance model as $J_{\|}^{\bmod }$. Comparing both $J_{\nabla \cdot E} / \Sigma_{\mathrm{P}}$ and $J_{\|}^{\bmod }$ to $J_{\|}$tests if the inclusion of an empirical conductance model can account for the gradient terms which are the suspected cause of the observed differences in the pre-midnight sector in Fig. 8.

Combining the $\boldsymbol{E}_{\perp}$ obtained from SuperDARN measurements with a tensor conductance according to Ohm's law for the Northern Hemisphere,

$$
\begin{aligned}
\boldsymbol{J}_{\perp}^{\mathrm{mod}} & =\bar{\Sigma} \cdot \boldsymbol{E}_{\perp} \\
& =\left(\begin{array}{cc}
\Sigma_{\mathrm{P}} & \Sigma_{\mathrm{H}} \\
-\Sigma_{\mathrm{H}} & \Sigma_{\mathrm{P}}
\end{array}\right) \cdot\left(\begin{array}{c}
E_{\theta} \\
E_{\phi}
\end{array}\right)
\end{aligned}
$$

$\boldsymbol{J}_{\|}^{\text {mod }}$ is obtained using Eq. 1 for $\boldsymbol{J}_{\perp}^{\mathrm{mod}}$ as

$$
J_{\|}^{\text {mod }}=\nabla \cdot J_{\perp}^{\text {mod }} .
$$

The ionospheric conductance model of Hardy et al. (1987) is parameterised by $K_{p}$ while the model of Rasmussen et al. (1988) has a solar flux (F10.7) dependence. These two conductance models were combined using $\Sigma_{\text {total }}=\sqrt{\Sigma_{\mathrm{PP}}^{2}+\Sigma_{\mathrm{EUV}}^{2}}$ to create an estimate of the Hall and Pedersen conductance that includes particle precipitation $\left(\Sigma_{\mathrm{PP}}\right)$ and solar EUV ( $\left.\Sigma_{\text {EUV }}\right)$ components. For the 1 November 2001 data, the conductance model was generated using $\mathrm{K}_{\mathrm{p}}=4.7$ and F10.7=235.6 sfu.
Using the SuperDARN data and Eq .(16), values for $J_{\|}^{\bmod }$ were calculated and are shown in Fig. 9. In the morning sector $J^{\text {mod }}$ shows a Region 1 (downward/blue) current at $70^{\circ}$ and 06:00 MLT. The location of this peak is shifted equatorward with respect to $J_{\nabla \cdot E} / \Sigma_{\mathrm{P}}$ and now matches the $J_{\|}$Region 1 current. This indicates the model conductance has represented conductance gradients in this region.

In the afternoon sector $J_{\|}^{\text {mod }}$ shows a R1/R2 system. With respect to $J_{\nabla \cdot E} / \Sigma_{\mathrm{P}}$, the $J^{\text {mod }} \mathrm{R} 1 / \mathrm{R} 2$ system has shifted approximately $1 \mathrm{~h}$ azimuthally towards midnight and poleward $\sim 2^{\circ}$ with the up-down boundary now located at $67^{\circ}$. Here the inclusion of gradient terms from a model conductance has not improved the agreement with $J_{\|}$. This result shows that model conductances together with SuperDARN convection maps, even in regions with radar returns, cannot reproduce the Birkeland currents derived from satellite magnetometer data. Hence, either the electric fields or the conductances are wrong, or most likely both. The R1/R2 type system seen between 12:00 and 15:00 MLT in $J_{\nabla \cdot E} / \Sigma_{\mathrm{P}}$ is not a significant feature in $J_{\|}^{\mathrm{mod}}$.

Hence we find that differences in the currents exist, even on the dayside where conductance gradients are expected to be small. Radar data are confined to the nightside for this event. It may be that differences in dayside currents, seen for example between $60^{\circ}-70^{\circ}$ around 09:00 MLT, are due to the statistical values used to constrain the SuperDARN data fit. The next event explores this possibility.

\subsection{Event 2: 3 October 2002, 13:00-14:00 UT}

The second event illustrates results with extensive dayside SuperDARN coverage. The interval is 13:00-14:00 UT on 3 October, 2002. The interplanetary parameters were checked for variability using data from the ACE spacecraft as shown in Fig. 10. The mean IMF parameters over 12:04-13:05 UT (56 min delay) were $B_{\mathrm{x}}=-2.4 \pm 0.8 \mathrm{nT}$, $B_{\mathrm{y}}=8.8 \pm 1.3 \mathrm{nT}, B_{\mathrm{z}}=-6.9 \pm 1.2 \mathrm{nT}, B_{\mathrm{t}}=11.5 \pm 0.3 \mathrm{nT}$, clock angle of $128 \pm 9^{\circ}, \mathrm{N}_{\mathrm{p}}=7.7 \pm 1.6 \mathrm{~cm}^{-3}, \mathrm{v}_{\mathrm{p}}=447 \pm 5 \mathrm{kms}^{-1}$ and $\mathrm{P}_{\mathrm{dyn}}=2.6 \pm 0.5 \mathrm{nPa}$. Conductance gradients in the ionosphere are expected to be less pronounced on the dayside as the conductance is dominated by solar EUV. The electric field data are shown in Fig. 11 for 3 October 2002 averaged over 13:00-14:00 UT. Most of the radar data were obtained between 11:00 and 17:00 MLT with some values also occurring pre-dawn. The electric potential is obtained as part of the spherical harmonic fitting process with data from the statistical model used where there are no radar returns (Ruohoniemi and Baker, 1998).

The magnetic field perturbations due to the Birkeland currents are shown in Fig. 12. During the one hour of this event, data from DMSP F13, F15 and Oersted, were available. In this case the Oersted and F13 tracks are close to each other. Since the passes occurred approximately $25 \mathrm{~min}$ apart, checking the vectors for alignment gives an extra indication of the stability of the currents during the integration 




Fig. 8. $J_{\nabla \cdot \boldsymbol{E}} / \Sigma_{\mathrm{P}}$ calculated from the divergence of the inertial electric field for 1 November, 2001 as shown in Fig. 7. The contours of $J_{\|}$obtained from the satellite data are overlayed for comparison. The shaded regions indicate where there were no radar returns. Note that the colour range differs from Fig. 6 due to the $\Sigma_{\mathrm{P}}^{-1}$ term.

time. From $70^{\circ}$ equatorward on the morning side both DMSP and Oersted show very good agreement. Near the geomagnetic pole the directions of the magnetic field values from F13 compared with Oersted differ but this is to be expected as the Iridium data adjacent to the two tracks shows a $90^{\circ}$ rotation in the direction of the field. At $70^{\circ}$ in the afternoon sector the two tracks are separated by approximately half an hour in MLT with both tracks exhibiting vectors consistent with a vortex centered on $72^{\circ}$ at 18:00 MLT. These features support the assumption of a stable current pattern over the time frame.

The Birkeland currents from the fitted satellite data are shown in Fig. 13. In the morning sector the R1/R2 system is clearly defined with an up-down current boundary at $65^{\circ}$ and 07:00 MLT. The Region 1 current (downward/blue) extends azimuthally through to noon. In the afternoon sector the $\mathrm{R} 1 / \mathrm{R} 2$ system has an up-down current boundary at $63^{\circ}$ and 17:00 MLT. The afternoon Region 1 current (upward/red) appears to extend through to 12:00 MLT. Inspection of the DMSP F15 particle energy spectra shows the spacecraft passed through the cusp at $\sim 71^{\circ}$ and 12:00 MLT. An additional up-down boundary is located at $63^{\circ}$ and 12:00 MLT.

These boundary locations may now be directly compared with those seen in $J_{\nabla \cdot E} / \Sigma_{\mathrm{P}}$. The div-E currents derived from SuperDARN data are shown Fig. 14. In the morning sector

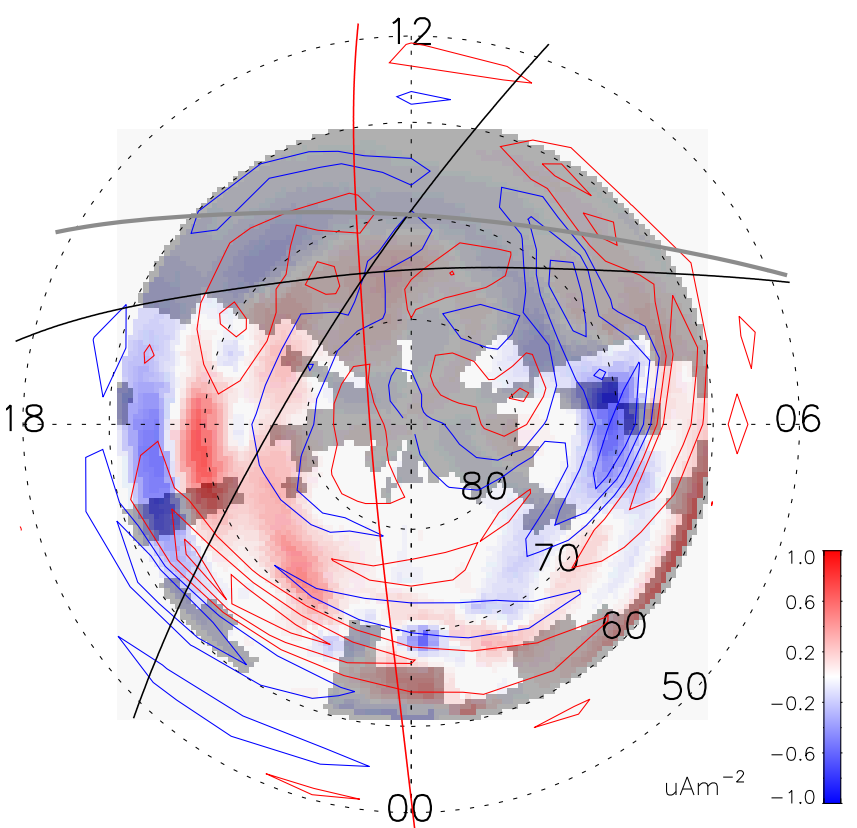

Fig. 9. $J_{\|}^{\text {mod }}$ calculated from the inertial electric field for 1 November, 2001 as shown in Fig. 7 and a conductance model (see text). The currents obtained from the satellite data are shown as contours and the shaded regions indicate where there were no radar returns. The DMSP and Oersted satellite tracks and sunlight terminator are also shown.

$J_{\nabla \cdot E} / \Sigma_{\mathrm{P}}$ shows a Region 1 current (downward/blue) near $76^{\circ}$ and between 02:00 and 08:00 MLT that may correspond with the $J_{\|}$Region 1 current near $70^{\circ}$. No Region 2 current is seen in $J_{\nabla \cdot E} / \Sigma_{\mathrm{P}}$ for the morning sector. Such a large difference appears to be due to the lack of SuperDARN observations here.

In the afternoon sector where SuperDARN observations are present, $J_{\nabla \cdot \boldsymbol{E}} / \Sigma_{\mathrm{P}}$ shows a R1/R2 system extending from 12:00 MLT through to 18:00 MLT. While this is consistent with $J_{\|}$, the up-down current boundaries are $3^{\circ}$ further poleward at 12:00 MLT and $3^{\circ}$ further equatorward at 17:00 MLT than observed for $J_{\|}$.

The model Birkeland current, $J^{\mathrm{mod}}$, calculated by combining SuperDARN data with a conductance model is shown in Fig. 15. The morning sector shows no significant current systems. The afternoon sector shows a R1/R2 system similar to $J_{\|}$. Relative to $J_{\nabla \cdot E} / \Sigma_{\mathrm{P}}$, the up-down current boundary at 17:00 MLT has been shifted poleward to be more consistent with $J_{\|}$indicating the conductance model contains reasonable gradients in this region. The difference in the up-down current boundary location between $J_{\|}^{\text {mod }}$ and $J_{\nabla \cdot E} / \Sigma_{\mathrm{P}}$ at noon is small $\left(\sim 1^{\circ}\right)$. 


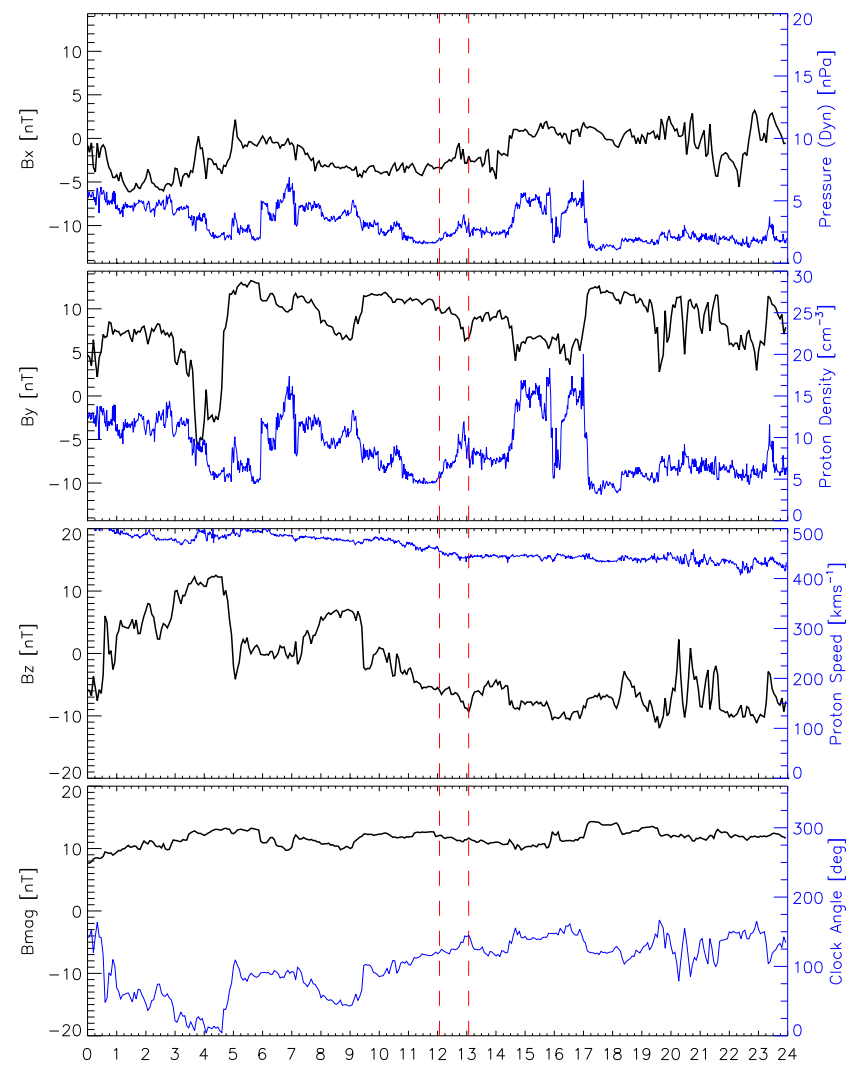

Fig. 10. ACE solar wind parameters showing several hours either side of 12:04-13:05 UT.

\section{Discussion}

The examples presented above show that disagreements in the location and direction of the Birkeland currents obtained from satellite magnetic field data and those obtained from radar data are not necessarily confined to either night or dayside regions. Both examples illustrate the finding that the satellite and radar derived currents disagree where there are no radar returns and the electric field calculation is constrained by the statistical model for convection. Where there is significant radar data coverage on the dayside, (e.g. 12:0015:00 MLT in Fig. 14), the approximation of $J_{\|}$by $J_{\nabla \cdot E}$ appears reasonable. However, there are also discrepancies where radar returns do exist. A closer examination of which parameter, $\boldsymbol{E}_{\perp}$ or $\nabla \Sigma$, contributes most to the disagreement is possible through an analysis of DMSP particle data. SuperDARN derived electric fields can be directly compared with those calculated from in-situ plasma drift velocity data available from the DMSP drift meter instruments. Furthermore, the magnitude of the conductance gradients may be directly estimated from DMSP particle precipitation observations.

The average electron energy, $E_{0}$, and electron energy flux, $I$, were obtained from the SSJ $/ 3$ particle detectors onboard the DMSP satellites and the Hall and Pedersen conduc-



Fig. 11. Electric field data (rotated by $90^{\circ}$ counter clockwise) derived from SuperDARN data averaged over the period 13:0014:00 UT, 3 October, 2002 with electric potential contours overlayed. Vectors are bold at locations where radar returns were received.

tance along the satellite track was estimated according to the method described by (Hardy et al., 1987). Combining these conductance estimates with $\boldsymbol{E}_{\perp}$ vectors from the radar data at the DMSP track locations using Eq. (3), we obtained estimates of the Birkeland currents. Therefore, estimates of the Birkeland currents along the DMSP tracks may be obtained from (i) the radar electric field and DMSP particle data based conductance estimates, $J_{\mathrm{SD}: \text { DMSP }}$, (ii) those derived from Iridium, DMSP and Oersted magnetic field data, $J_{\|}$, and (iii) the radar electric field and empirical model conductance estimates, $J_{\|}^{\mathrm{mod}}$.

The conductances along the DMSP tracks $\left(\Sigma^{\text {DMSP }}\right)$ were calculated using the particle precipitation (PP) contribution as (Hardy et al., 1987)

$$
\begin{aligned}
& \Sigma_{\mathrm{PP}: \mathrm{P}}^{\mathrm{DMSP}}=\left[40 E_{0} /\left(16+E_{0}^{2}\right)\right] I^{0.5} \\
& \Sigma_{\mathrm{PP}: \mathrm{H}}^{\mathrm{DMSP}}=0.45\left(E_{0} / 1 \mathrm{keV}\right)^{5 / 8} \Sigma_{\mathrm{PP}: \mathrm{P}}^{\mathrm{DMSP}}
\end{aligned}
$$

and the EUV contribution from the model of Rasmussen et al. (1988) combined using the approximation presented in Sect. 4.1. The spatial information in conductance is limited to the along track direction with incomplete knowledge of the $\nabla \Sigma$ terms in Eq. (3). To best utilise the available data it was assumed that terms involving $\frac{\partial}{\partial \phi}$ (variations with MLT) would be much smaller compared with $\frac{\partial}{\partial \theta}$ (variations with 


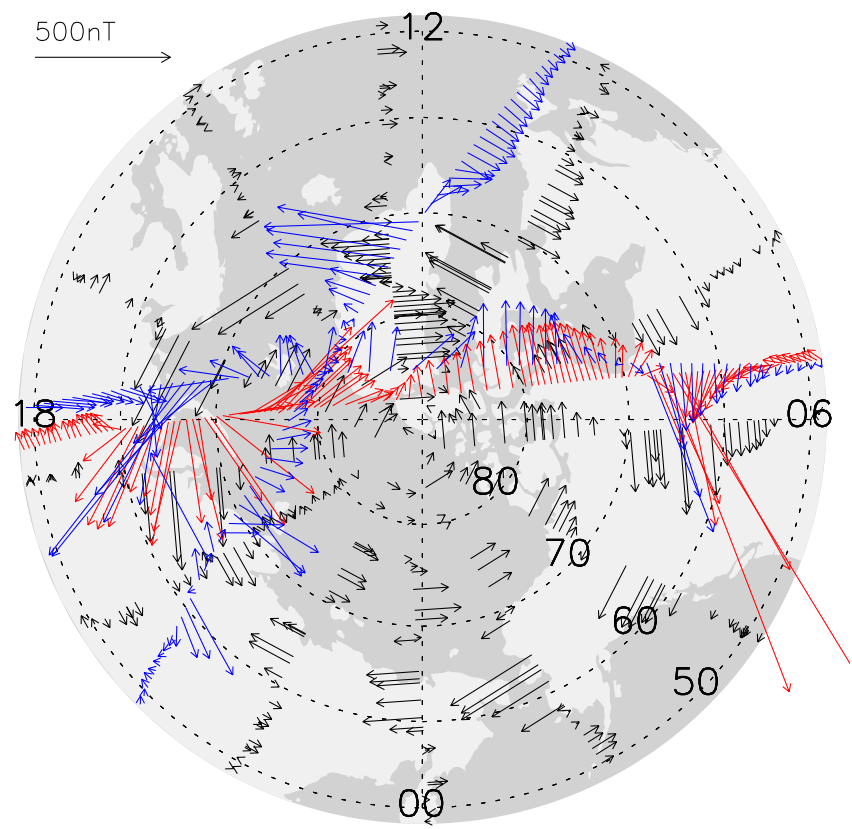

Fig. 12. Cross-track component of the Iridium $\triangle \boldsymbol{b}$ data (black) for 3 October 2002, 13:00-14:00 UT. The red track represents full vector $\Delta \boldsymbol{b}$ observations from the Oersted satellite pass for 13:38-14:08 UT while the two blue tracks are DMSP satellites F13 (dusk-dawn) and F15 for the intervals 13:13-13:44 UT and 13:3414:05 UT, respectively.

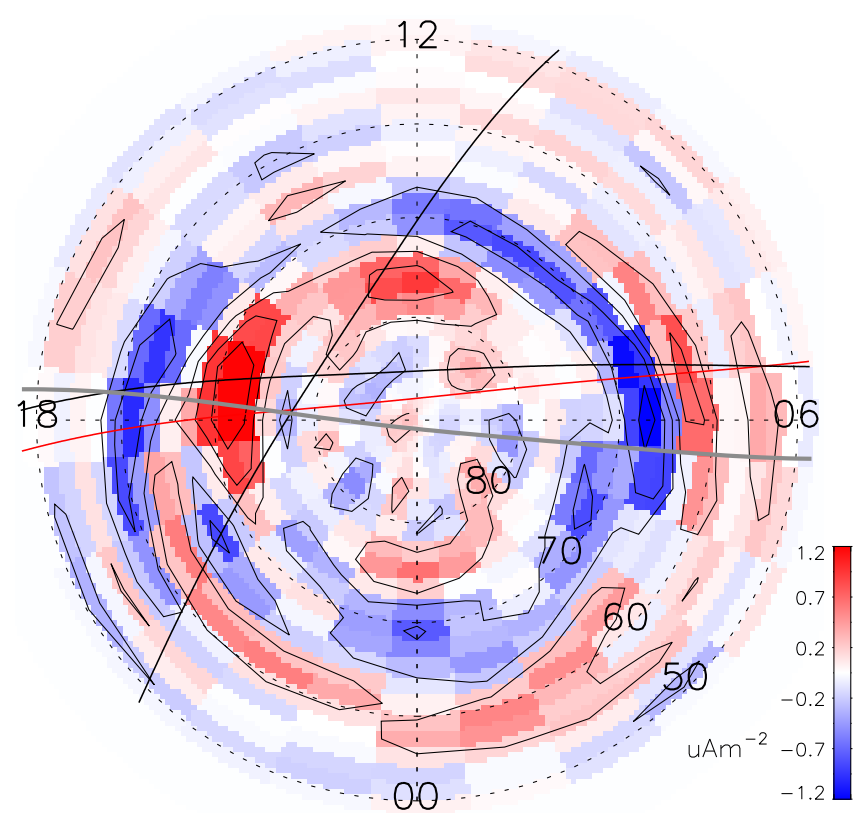

Fig. 13. Birkeland currents, $J_{\|}$calculated from the satellite magnetic perturbation data for 3 October, 2003 as shown in Fig. 12 using Eq. (12). The parameters used in the SCHA were $\theta_{c}=50^{\circ}, k=15$ and $m=5$.

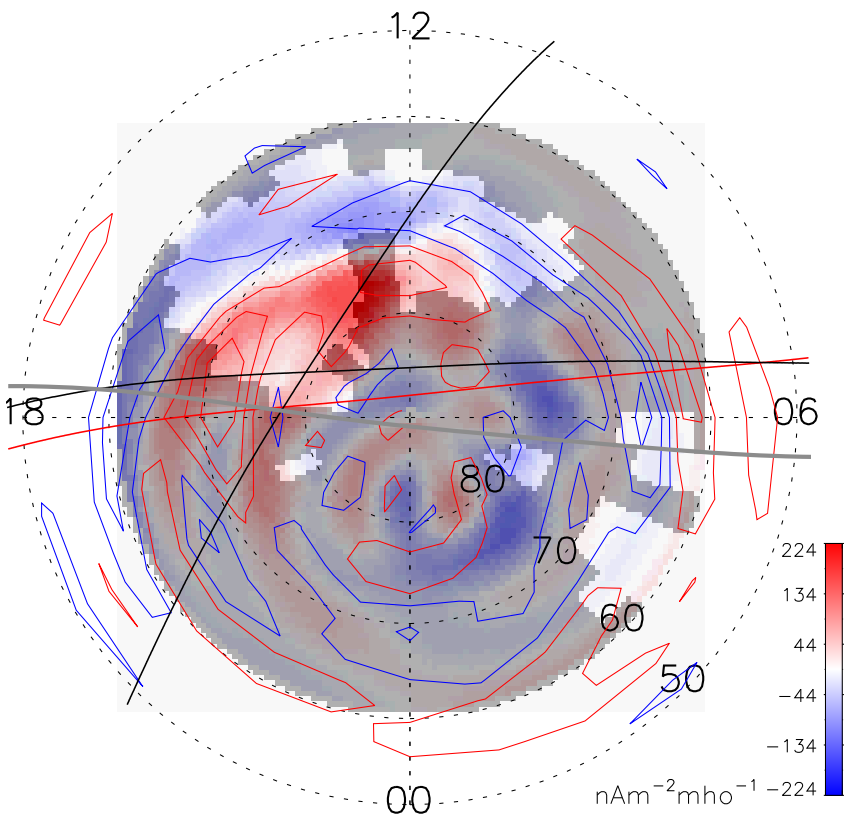

Fig. 14. $J_{\nabla \cdot E} / \Sigma_{\mathrm{P}}$ calculated from the divergence of the electric field for 3 October, 2003 as shown in Fig. 11 with $J_{\|}$contours overlayed for comparison. Shaded regions indicate no radar coverage. Note that the colour range differs from Fig. 13 due to the $\Sigma_{\mathrm{P}}^{-1}$ term.

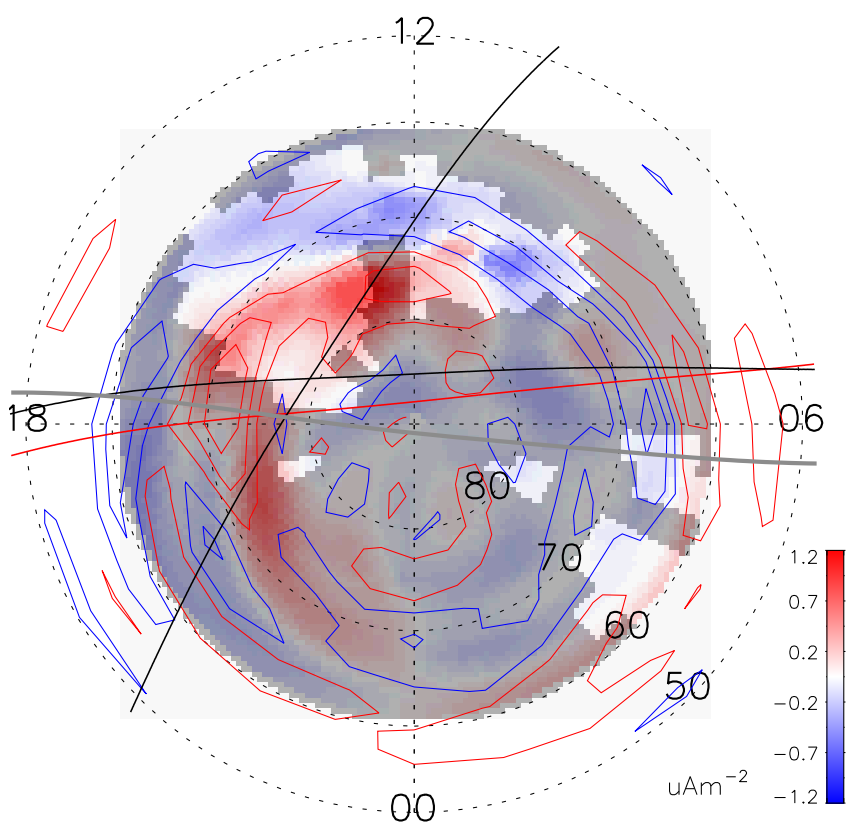

Fig. 15. $J^{\text {mod }}$ obtained from the radar electric field data for 3 October, 2003 as shown in Fig. 11 and the conductance model (see text). The contours of the currents, $J_{\|}$obtained from the satellite data are overlayed and shaded regions indicate no radar coverage. 
latitude) terms. The spatial resolution of the DMSP conductance data was reduced to SuperDARN and Iridium spatial scales by applying a low-pass filter with the cut-off spatial size set at $3^{\circ}$ in latitude.

Figure 16 shows the various contributions to the conductance and current evaluated along the DMSP F13 track for the 1 November 2001 data (cf. Figs. 4-9). The seven panels detail the various electrodynamic parameters and how they vary across the Birkeland current system. Panel 1 shows the Birkeland current derived from the three separate methods. These are (i) the combined Iridium, Oersted and DMSP magnetic field perturbation data as described in Sect. 3 (black; $J_{\|}$), (ii) combining the conductance data estimated from DMSP particle data with the SuperDARN derived $\boldsymbol{E}_{\perp}$ (blue; $J_{\mathrm{SD}: \mathrm{DMSP}}$ ) and (iii) the divergence of the electric fields from radar measurements with the conductance model (green; $J^{\text {mod }}$ ). On the right hand side of panel 1 is the axis for $J_{\nabla \cdot E} / \Sigma_{\mathrm{P}}(\mathrm{red})$, the divergence of the electric field estimated from the radar data.

Panel 2 in Fig. 16 shows the individual terms for the current as described by Eq. (3) and using the DMSP conductance data. These are the terms used to construct the blue curve in panel 1. Panel 3 shows a vector representation of $\boldsymbol{E}_{\perp}$ as derived from both SuperDARN observations (black; $\boldsymbol{E}_{\perp}$ ) and DMSP drift meter data (red; $\boldsymbol{E}_{\perp}^{\text {DMSP }}$ ). Panel 4 shows the gradients in the Hall and Pedersen conductances obtained from the DMSP particle data (Eqs. 17, 18) with latitude as well as the full gradient terms available from the model conductance. Panel five shows the satellite MLT coordinate. Panels 6 and 7 show the Pedersen and Hall conductances estimated from the DMSP particle data (blue; $\Sigma_{\mathrm{P}}^{\mathrm{DMSP}}, \Sigma_{\mathrm{H}}^{\mathrm{DMSP}}$ ). The filtered conductances are shown in black. These are compared with values from the conductance model (green; $\left.\Sigma_{\mathrm{P}}^{\text {mod }}, \Sigma_{\mathrm{H}}^{\bmod }\right)$. All panels are plotted versus satellite position in colatitude with the direction of satellite travel from left to right. Negative (-ve) colatitude values are used to plot the full track. The shaded parts indicate sections of the satellite tracks where the angle between the north-south direction and the satellite track is greater than $45^{\circ}$. Panel one, for all figures except Fig. 16, includes a solid black bar indicating the regions where there are radar returns.

The path of DMSP F13 in Fig. 16 is from 17:00 MLT to 07:30 MLT. At the start of the data segment, which is during the afternoon sector, there is very little conductance due to particle precipitation as shown by panels 6 and 7. The resultant conductance gradient terms, as shown in panel 2, are very small giving almost no Birkeland current. The estimates of $J_{\|}$in panel 1 (black) show a small upward current at $-23^{\circ}$. The current calculated from $\Sigma_{\mathrm{P}} \boldsymbol{\nabla} \cdot \boldsymbol{E}_{\perp}$ in panel 2 (black) is a poor estimate of the Birkeland currents. This may be expected in regions where SuperDARN returns are not available. Later on the dawn side, there are considerable conductance enhancements due to particle precipitation that correspond with the R1/R2 current system seen in $J_{\|}$(Fig. 6). Panel 4 in Fig. 16 shows larger conductance gradients com- pared with the afternoon sector but a small SuperDARN electric field equatorward of $20^{\circ}$, resulting in negligible current terms in panel 2 and the small $J_{\text {SD:DMSP }}$ current (blue) in panel 1. The downward current observed by Iridium at $21^{\circ}$ is not duplicated by any of the other methods which indicates an incorrect $\boldsymbol{E}_{\perp}$ where there are no radar data. Therefore, the statistical electric potential model used to constrain the radar data does not reproduce the appropriate Birkeland currents in the morning sector for this day. This point is further illustrated by the comparison of $\boldsymbol{E}_{\perp}$ and $\boldsymbol{E}_{\perp}^{\mathrm{DMSP}}$ in panel 3 where the SuperDARN statistical model predicts negligible electric field strength while DMSP shows large values with a clear field reversal near $20^{\circ}$.

The DMSP F15 satellite for the 1 November 2001 event passes directly across the large currents observed between $20^{\circ}$ and $30^{\circ}$ at 21:00 MLT. Figure 17 shows the associated conductance enhancements and gradient terms in panels 2 and 4. Consider the $J_{\text {SD:DMSP }}$ (blue) and $J_{\nabla \cdot E} / \Sigma_{\mathrm{P}}$ (red) curves in the negative colatitude section of Fig. 17, panel 1. This is a case where the conductance gradient terms are comparable or larger compared with the $\Sigma_{\mathrm{P}} \boldsymbol{\nabla} \cdot \boldsymbol{E}_{\perp}$ estimate from the radar data. Conductance gradients are expected to be more pronounced on the nightside where solar EUV induced conductance does not occur. In panel 2, the conductance gradients derived from the DMSP particle data produce large grad-Sigma current terms and are effective in adjusting the radar estimate of the currents to be almost equal to $J_{\|}$(black) in panel 1. The rather poor estimates of the current based on the conductance model $\left(J_{\|}^{\mathrm{mod}}\right)$ are shown by the green curve in panel 1 . In panel 1 near $-27^{\circ}, J_{\text {SD:DMSP }}$ (blue) is shifted poleward by $\sim 3^{\circ}$ compared with the satellite magnetic field based estimates of the current $\left(J_{\|}\right.$; black). Furthermore, the large negative current here is not reproduced in $J_{\text {SD:DMSP }}$. In this case the radar data do not extend far enough equatorward, missing electric field information in regions where the conductance gradients are substantial as seen in panel 4 of Fig. 17. Panel 3 shows a large southward $\boldsymbol{E}_{\perp}^{\text {DMSP }}$ near $-30^{\circ}$ whereas the SuperDARN estimate tends to zero here, even with considerable radar coverage. The dayside portion of the F15 track shows a large electric field but negligible conductances up to $23^{\circ}$ and some small Hall conductance enhancements but small electric field magnitudes past $23^{\circ}$ resulting in negligible Birkeland current magnitudes. There is also a large discrepancy between the SuperDARN statistical model $\boldsymbol{E}_{\perp}$ and $\boldsymbol{E}_{\perp}^{\mathrm{DMSP}}$ as shown in the positive colatitudes of panel 3 .

Data for the path of DMSP F13 for 3 October, 2002 is shown in Fig. 18. The F13 track is dusk to dawn, on the dayside of the sunlit terminator, as shown in Fig. 13. Conductance enhancements are observed in both the afternoon and morning sectors. The afternoon sector data (-ve colatitude) in panel 2 of Fig. 18 indicates the $\Sigma_{\mathrm{P}} \boldsymbol{\nabla} \cdot \boldsymbol{E}_{\perp}$ term represents most of the Birkeland current poleward of $25^{\circ}$ while equatorward of this, coinciding with the Region 2 current, the term 


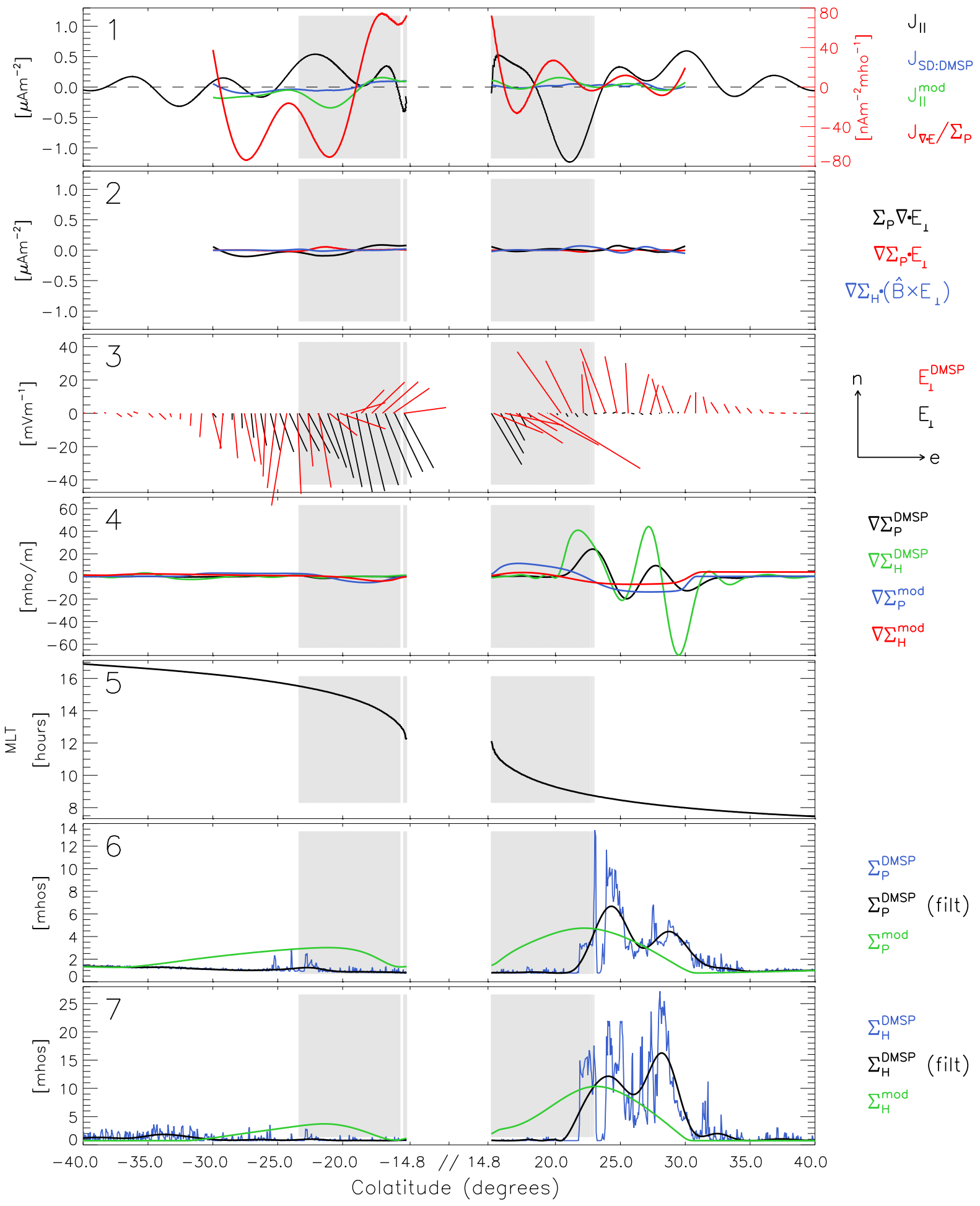

Fig. 16. Iridium, DMSP F13, and radar data for 1 November, 2001. Panel 1: Birkeland currents estimated from various sources (see text), Panel 2: Birkeland current terms from Eq. (3) using DMSP F13 derived conductance, Panel 3: Electric field from the radars and DMSP drift meter data, Panel 4: DMSP F13 and model derived conductance gradients, Panel 5: DMSP satellite MLT coordinate, Panel 6: Pedersen conductance derived from the DMSP F13 data and the conductance model, panel 7: Hall conductance derived from the DMSP F13 data and the conductance model. The lack of a black bar in panel 1 indicates no radar coverage along this track. 


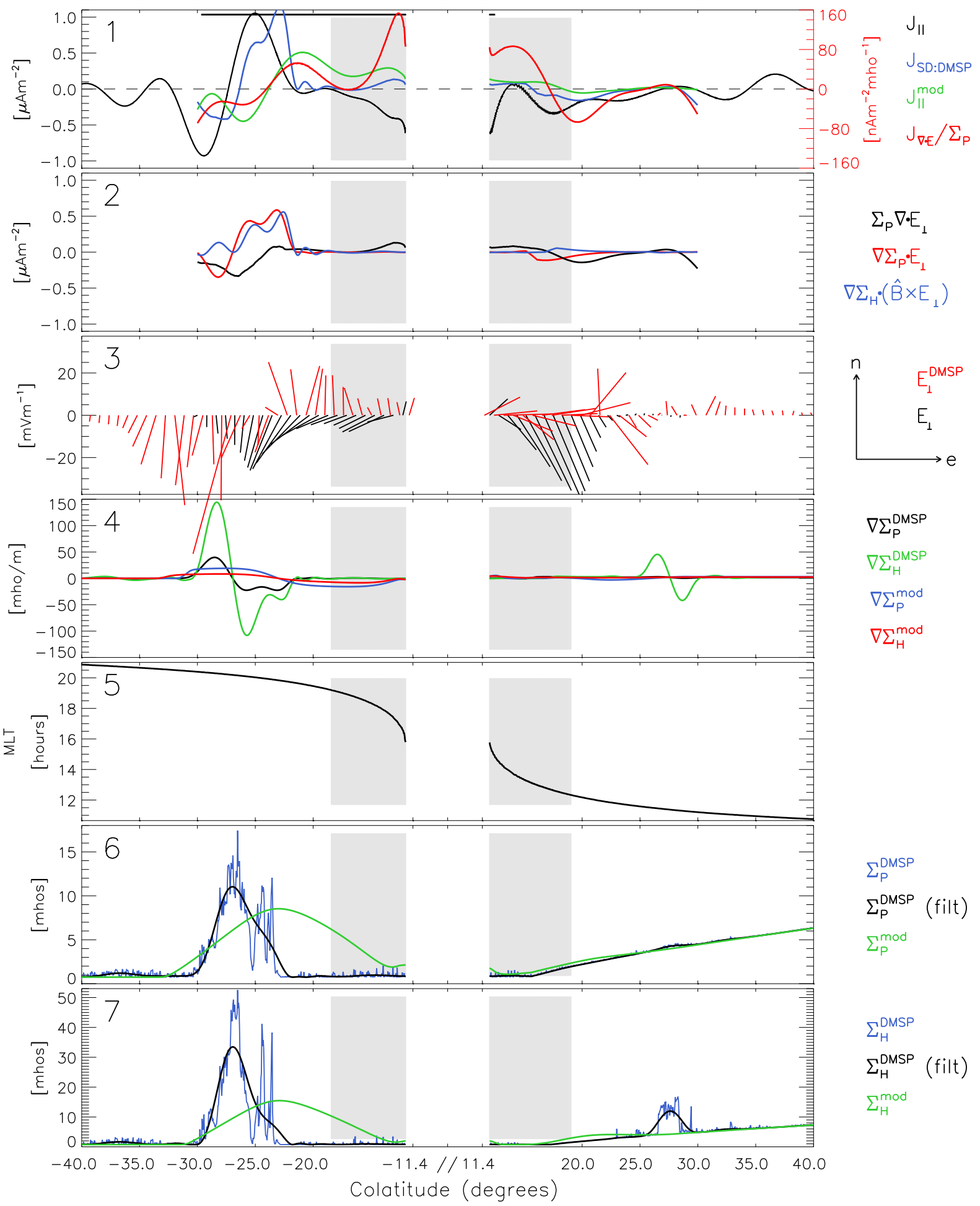

Fig. 17. Iridium, DMSP F15, and radar data for 1 November, 2001. Panel 1: Birkeland currents estimated from various sources (see text), Panel 2: Birkeland current terms from Eq. (3) using DMSP F15 derived conductance, Panel 3: Electric field from the radars and DMSP drift meter data, panel 4: DMSP F15 and model derived conductance gradients, Panel 5: DMSP satellite MLT coordinate, panel 6: Pedersen conductance derived from the DMSP F15 data and the conductance model, panel 7: Hall conductance derived from the DMSP F15 data and the conductance model. 


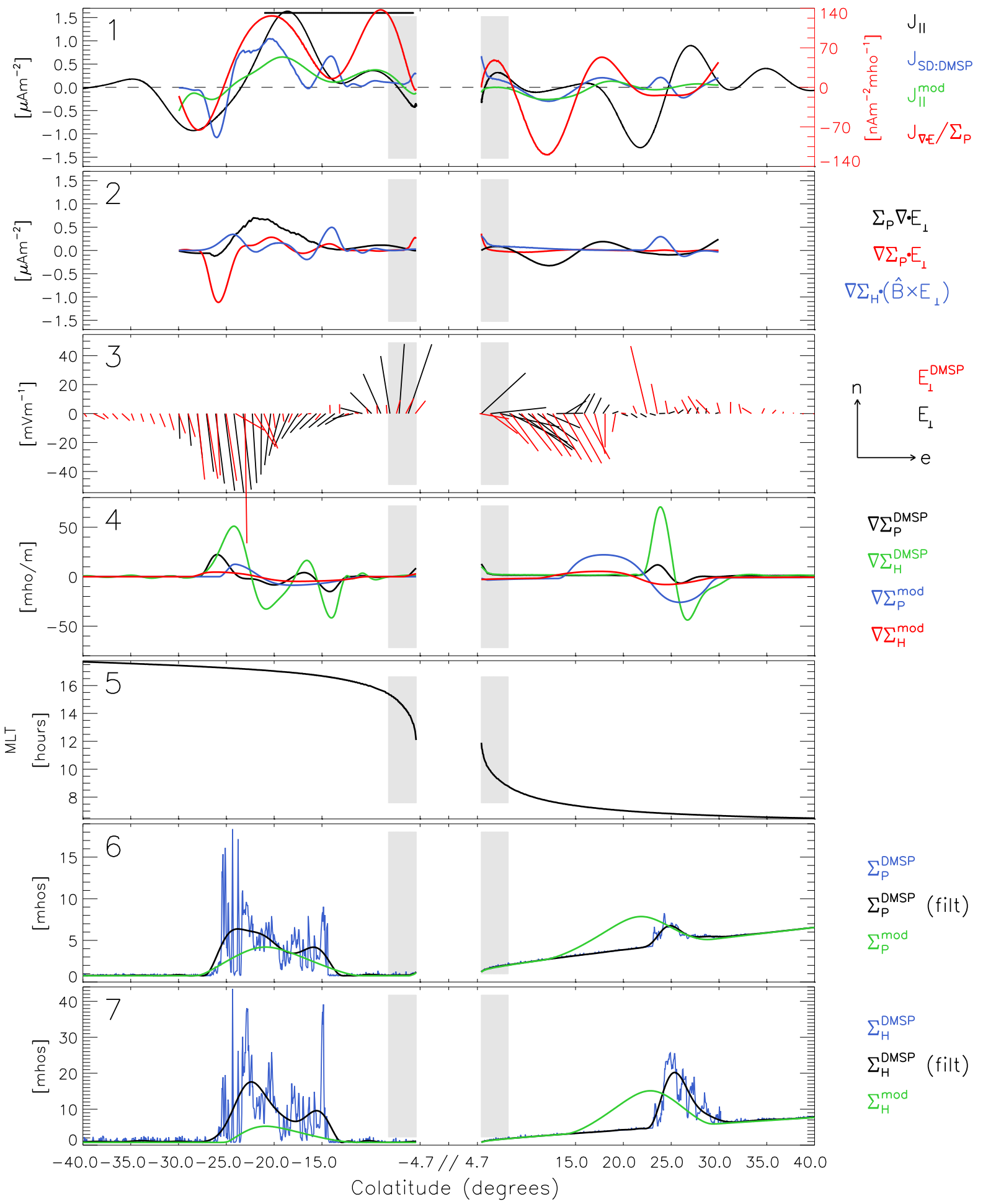

Fig. 18. Iridium, DMSP F13, and radar data for 3 October, 2002. Panel 1: Birkeland currents estimated from various sources (see text), panel 2: Birkeland current terms from Eq. (3) using DMSP F13 derived conductance, panel 3: Electric field from the radars and DMSP drift meter data, panel 4: DMSP F13 and model derived conductance gradients, panel 5: DMSP satellite MLT coordinate, panel 6: Pedersen conductance derived from the DMSP F13 data and the conductance model, panel 7: Hall conductance derived from the DMSP F13 data and the conductance model. 


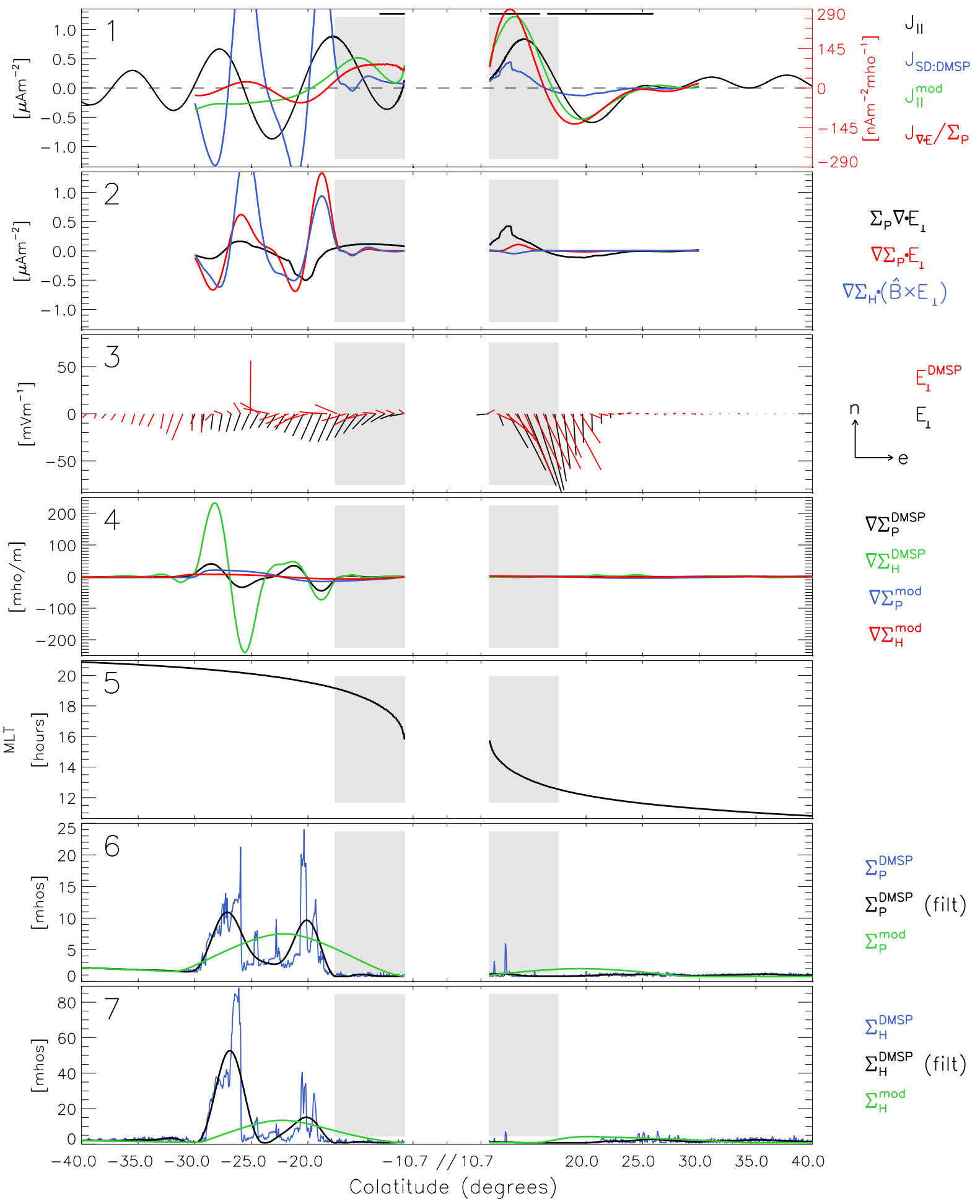

Fig. 19. Iridium, DMSP F15, and radar data for 3 October, 2002. Panel 1: Birkeland currents estimated from various sources (see text), panel 2: Birkeland current terms from Eq. 3 using DMSP F15 derived conductance, panel 3: Electric field from the radars and DMSP drift meter data, panel 4: DMSP F15 and model derived conductance gradients, panel 5: DMSP satellite MLT coordinate, panel 6: Pedersen conductance derived from the DMSP F15 data and the conductance model, panel 7: Hall conductance derived from the DMSP F15 data and the conductance model. 
involving the gradient in Pedersen conductance dominates. Comparing $J_{\nabla \cdot E} / \Sigma_{\mathrm{P}}$ (panel 1; red), with the Birkeland current estimates from satellite data $\left(J_{\|}\right.$; black) in panel 1 shows a $3^{\circ}$ offset in the R1/R2 up-down current boundary. Inclusion of the gradient terms in panel 2 is shown by $J_{\text {SD:DMSP }}$ (blue) in panel 1 where there is little change in the R1/R2 boundary location. The morning sector data from the F13 track shows particle induced conductance enhancements between $23^{\circ}$ and $30^{\circ}$ colatitude with associated gradients shown in panel 4. While gradients of the Pedersen and Hall conductances exist, panel 2 shows negligible currents from the grad-Sigma terms due to small electric field values. As a result, $J_{\mathrm{SD} \text { :DMSP }}$ completely misses the Region 1 and 2 currents detected by the satellite magnetometer data. From Fig. 11 there are sparse SuperDARN observations in this region and panel 3 shows that the SuperDARN statistical model poorly represents $\boldsymbol{E}_{\perp}^{\mathrm{DMSP}}$.

The data from DMSP F15 for 3 October, 2002 is shown in Fig. 19. The satellite tracks from pre midnight through to pre-noon, passing close to $80^{\circ}$ latitude around 16:00 MLT. SuperDARN data were available for the afternoon segment, although near 16:00 MLT, F15 is tracking azimuthally, providing poor estimates of the latitudinal derivatives in conductance. Begining with the start of the track, on the nightside of the sunlit terminator (-ve colatitude), the DMSP derived conductance gradients and associated currents shown in panels 2 and 4 of Fig. 19 reflect the triple current system. In this case the grad-Sigma terms are the dominant contributions to $J_{\text {SD:DMSP }}$ and therefore show considerable deviation from the div-E component (red; panel 1). $J_{\text {SD:DMSP }}$ (panel 1; blue) shows Birkeland current estimates somewhat similar in shape and magnitude to that of $J_{\|}$(black). However, differences in the latitudes where these currents are zero become larger at lower latitudes. This latitudinal offset is expected to be related to the lack of SuperDARN returns in this region. On the dayside portion of the F15 track, the conductance gradient terms are small (panel 2), as expected, and $J_{\text {SD:DMSP }}$ is also small. Considering the agreement between $\boldsymbol{E}_{\perp}$ and $\boldsymbol{E}_{\perp}^{\text {DMSP }}$ shown in panel 3, it appears the smoothed nature of the SuperDARN fit is the cause of a small Region 2 current in $J_{\text {SD:DMSP }}$. The model conductance based estimates, $J_{\|}^{\bmod }$ (green) agree quite well, a fortuitous result considering the agreement between DMSP and model conductance is poor in all panels 6 and 7 .

\section{Conclusions}

The first comparison of div-E currents derived from SuperDARN observations with Birkeland currents from satellite data over the entire auroral oval and polar cap has been presented. The results show that the distribution of large-scale region 1 and 2 current systems can be represented in regions of extensive radar returns. However, agreement with satellite derived currents was obtained only for the event for sunlit ionosphere conditions, i.e., when the ionospheric conductance was dominated by solar EUV. In regions where the spatial coverage of SuperDARN returns is sparse or absent, the div-E current estimates do not agree with Birkeland currents from satellite data. The div-E approach also did not work for the nightside case in darkness even though radar returns spanned the region of interest. The results suggest that the model electric potential used to constrain the SuperDARN estimate of the ionospheric electric field does not adequately represent the electric field in regions without radar returns for these cases. Moreover, for the nightside case, the lack of agreement even including model conductances implies that either the conductances or the electric field estimates or both do not represent actual conditions associated with the observed Birkeland currents.

Under the conditions of radar coverage and small ionospheric conductance gradients (e.g., Fig. 19) the SuperDARN based div-E and satellite estimates show agreement in the locations of boundaries between upward and downward currents to within $3^{\circ}$ latitude. Details on scale size smaller than $\sim 3^{\circ}$ cannot be resolved with Iridium data.

In regions where the satellite data show conductance gradients, the grad-Sigma contribution to the total current may be considerably larger than the div-E component (e.g. Fig. 19, -ve colatitude). Also, in regions where there are both conductance gradients and radar coverage, including the grad-Sigma terms based upon in-situ estimates of conductance can considerably improve the agreement with Birkeland currents obtained from satellite data (e.g., Fig. 17). The inclusion of an empirical conductance model to the radar data does not account for the currents produced by the grad-Sigma terms. Therefore, combining a conductance model, such as the one employed here, with the global SuperDARN derived electric field data does not necessarily improve the estimation of the Birkeland currents from the radar data.

\section{Appendix A}

\section{Non-integral associated Legendre functions}

Analytical expression for the non-integral (real n, integral m) associated Legendre functions in terms of the hypergeometric function $F$ (Hobson, 1955)

$$
\begin{aligned}
P_{n}^{m}(\cos \theta)=(-1)^{m} & \frac{\sin ^{m} \theta}{2^{m} \Gamma(m+1)} \frac{\Gamma(n+m+1)}{\Gamma(n-m+1)} \\
& F([m-n, n+m+1] ; m+1 ; \mu)
\end{aligned}
$$


and the theta derivative

$$
\frac{\partial}{\partial \theta} P_{n}^{m}(\cos \theta)=(-1)^{m} \frac{\sin ^{m} \theta}{2^{m} \Gamma(m+1)} \frac{\Gamma(n+m+1)}{\Gamma(n-m+1)}
$$

$$
\begin{aligned}
& \left(\frac{\sin \theta(m-n)(n+m+1)}{2(m+1)}\right. \\
& F([m+1-n, n+m+2] ; m+2 ; \mu) \\
& \left.+\frac{m \cos \theta}{\sin \theta} F([m-n, n+m+1] ; m+1 ; \mu)\right)
\end{aligned}
$$

where $\mu=\frac{1-\cos \theta}{2}$.

Acknowledgements. This research was supported by grants from the University of Newcastle and the Cooperative Research Centre for Satellite Systems under the Australian government Cooperative Research Center (CRC) scheme. We thank Iridium Satellite LLC for providing the engineering magnetometer data. We thank the ACE team for providing solar wind data, F. Rich for providing the DMSP magnetometer data and Ma. Hairston for the DMSP particle data. The Oersted magnetometer data was kindly made available by F. Christiansen. Support for analysis of the Iridium data was provided by NSF grants ATM-0334668 and ATM-0101064.

Topical Editor M. Pinnock thanks O. Amm and P. Stauning for their help in evaluating this paper.

\section{References}

Amm, O.: Method of characteristics for calculating ionospheric electrodynamics from multisatellite and ground-based radar data, J. Geophys. Res., 107, 1270-1281, 2002.

Amm, O., Aksnes, A., Stadsnes, J., Østgaard, N., Vondrak, R. R., Germany, G. A., Lu, G., and Viljanen, A.: Mesoscale ionospheric electrodynamics of omega bands determined from ground-based electrodynamic and satellite optical observations, Ann. Geophys., 23, 325-342, 2005.

Anderson, B. J., Takahashi, K., and Toth, B. A.: Sensing global Birkeland currents with Iridium engineering magnetometer data, Geophys. Res. Lett., 27, 4045-4048, 2000.

Backus, G.: Poloidal and Toroidal Fields in Geomagnetic Field Modeling, Reviews of Geophysics, 24, 75-109, 1986.

Birkeland, K.: The Norwegian Aurora Polaris Expedition 19021903, Vol. 1, H. Aschehoug \& Co., Christiania, Norway, 1908.

Cowley, S. W. H.: Magnetosphere-Ionosphere Interactions: A Tutorial Review, in Magnetospheric Current Systems, edited by: Ohtani, S., Fujii, R., Hesse, M., and Lysak, R. L., Geophysical Monograph 118, American Geophysical Union, Washingtion, D.C., pp. 91-106,2000.

de Santis, A.: Conventional Spherical Harmonic analysis for regional modelling of the geomagnetic field, Geophys. Res. Lett., 19, 1065-1067, 1992.

Dunlop, M. W., Balogh, A., Glassmeier, K. H., and Robert, P.: Fourpoint Cluster application of magnetic field analysis tools: The Curlometer, J. Geophys. Res., 107, 1384-1397, 2002.

Eriksson, S., Bonnell, J. W., Blomberg, L. G., Ergun, R. E., Marklund, G. T., and Carlson, C. W.: Lobe cell convection and fieldaligned currents poleward of the region 1 current system, J. Geophys. Res., 107, 1185-1191, 2002.
Greenwald, R. A., Baker, K. B., Hutchins, R. A., and Hanuise, C.: An HF phased-array radar for studying small-scale structure in the high-latitude ionosphere, Radio Sci., 20, 63-79, 1985.

Greenwald, R. A., Baker, K. B., Dudeney, J. R., Pinnock, M., Jones, T. B., Thomas, E. C., Villain, J.-P., Cerisier, J.-C., Senior, C., Hanuise, C., Hunsucker, R. D., Sofko, G., Koehler, J., Nielsen, E., Pellinen, R., Walker, A. D. M., Sato, N., and Yamagishi, H.: Darn/SuperDARN: A global view of the dynamics of highlatitude convection, Space Sci. Rev., 71, 761-796, 1995.

Haines, G. V.: Spherical Cap Harmonic Analysis, J. Geophys. Res., 90, 2583-2591, 1985.

Haines, G. V.: Computer Programs for spherical cap harmonic analysis of potential and general fields, Computers and Geosciences, 14, 413-447, 1988.

Hardy, D. A., Gussenhoven, M. S., Raistrick, R., and McNeil, W. J.: Statistical and Functional Representations of the Pattern of Auroral Energy Flux, Number Flux, and Conductivity, J. Geophys. Res., 92, 12 275-12 294, 1987.

Hobson, E. W.: The Theory of Spherical and Ellipsoidal Harmonics, Chelsea Publishing Company, New York, 1955.

Iijima, T. and Potemra, T. A.: The Amplitude distribution of fieldaligned currents at northern high latitudes observed by Triad, J. Geophys. Res., 81, 2165-2174, 1976.

Iijima, T., Potemra, T. A., Zanetti, L. J., and Bythrow, P. F.: Large-scale Birkeland currents in the dayside polar region during stringly northward IMF: A new Birkeland current system, J. Geophys. Res., 89, 7441-7452, 1984.

Kamide, Y., Richmond, A. D., and Matsushita, A.: Estimation of Ionospheric Electric Fields, Ionospheric Currents, and FieldAligned Currents from Ground Magnetic Records, J. Geophys. Res., 86, 801-813, 1981.

Kelley, M. C.: The Earth's Ionosphere, Plasma Physics and Electrodynamics, Academic Press, Inc., San Diego, California, 1989.

Korth, H., Anderson, B. J., Frey, H. U., and Waters, C. L.: Highlatitude electromagnetic and particle energy flux during an event with sustained strongly northward IMF, Ann. Geophys., 23, 1295-1310, 2005.

Kosch, M. J., Scourfield, M. W. J., and Amm, O.: The importance of conductivity gradients in ground-based field-aligned current studies, Adv. Space Res., 27, 1277-1282, 2001.

Kustov, A. V., Lyatsky, W. B., Sofko, G. J., and Xu, L.: FieldAligned currents in the polar cap at small IMF $\mathrm{B}_{Z}$ and $\mathrm{B}_{Y}$ inferred from SuperDARN radar observations, J. Geophys. Res., 105, 205-214, 2000.

McWilliams, K. A., Yeoman, T. K., Sigwarth, J. B., Frank, L. A., and Brittnacher, M.: The dayside ultraviolet aurora and convection responses to a southward turning of the interplanetary magnetic field, Ann. Geophys., 19, 707-721, 2001.

Moshier, S. L.: Cephes Math Library, http://www.netlib.org/ cephes/, 1995

Olsen, N.: Ionospheric F region currents at middle and low latitudes estimated from Magsat data, J. Geophys. Res., 102, 4563-4576, 1997.

Rasmussen, C. E., Schunk, R. W., and Wickwar, V. B.: A Photochemical Equilibrium Model for Ionospheric Conductivity, J. Geophys. Res., 93, 9831-9840, 1988.

Richmond, A. D. and Kamide, Y.: Mapping electrodynamic features of the high-latitude ionosphere from localized observations - Technique, J. Geophys. Res., 93, 5741-5759, 1988. 
Richmond, A. D. and Thayer, J. P.: Ionospheric Electrodynamics: A Tutorial, in Magnetospheric Current Systems, edited by S. Ohtani, R. Fujii, M. Hesse, and R. L. Lysak, Geophysical Monograph 118, American Geophysical Union, Washingtion, D.C., pp. 131-146,2000.

Ruohoniemi, J. M. and Baker, K. B.: Large-scale imaging of highlatitude convection with Super Dual Auroral Radar Network HF radar observations, J. Geophys. Res., 103, 20 797-20 811, 1998.

Sato, M., Kamide, Y., Richmond, A. D., Brekke, A., and Nozawa, S.: Regional estimation of electric fields and currents in the polar ionosphere, Geophys. Res. Lett., 22, 283-286, 1995.

Shepherd, S. G. and Ruohoniemi, J. M.: Electrostatic potential patterns in the high-latitude ionosphere constrained by SuperDARN measurements, J. Geophys. Res., 105, 23 005-23 014, 2000.

Sofko, G. J., R.Greenwald, and Bristow, W.: Direct determination of large-scale magnetospheric field-aligned currents with SuperDARN, Geophys. Res. Lett., 22, 2041-2044, 1995.

Stauning, P.: Field-aligned ionospheric current systems observed from Magsat and Oersted satellites during northward IMF, Geophys. Res. Lett., 29, 8005-8008, 2002.
Untiedt, J. and Baumjohann, W.: Studies of polar current systems using the IMS Scandinavian Magnetometer Array, Space Science Reviews, 63, 245-390, 1993.

Waters, C. L., Anderson, B. J., and Liou, K.: Estimation of Global Field Aligned Currents Using the Iridium System Magnetometer Data, Geophys. Res. Lett., 28, 2165-2168, 2001.

Waters, C. L., Anderson, B. J., Greenwald, R. A., Barnes, R. J., and Ruohoniemi, J. M.: High-latitude poynting flux from combined Iridium and SuperDARN data, Ann. Geophys., 22, 2861-2875, 2004.

Weimer, D. R.: A new technique for the mapping of ionospheric field-aligned currents from satellite magnetometer data, in: Magnetospheric Current Systems, edited by: Ohtani, S., Fujii, R., Hesse, M., and Lysak, R. L., Geophysical Monograph 118, American Geophysical Union, Washingtion, D.C., pp. 381-388, 2000.

Zanetti, L. J., Baumjohann, W., and Potemra, T. A.: Ionospheric and Birkeland Current Distributions Inferred From the MAGSAT Magnetometer Data, J. Geophys. Res., 88, 4875-4884, 1983.

Zmuda, A. J., Martin, J. H., and Heuring, F. T.: Transverse Magnetic Disturbances at 1100 Kilometers in the Auroral Region, J. Geophys. Res., 71, 5033-5045, 1966. 